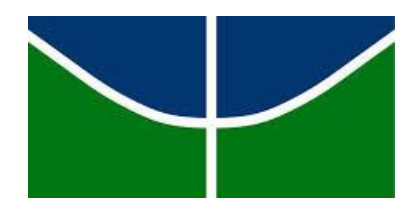

UNIVERSIDADE DE BRASÍLIA

INSTITUTO DE CIÊNCIAS EXATAS

DEPARTAMENTO DE MATEMÁTICA

\title{
Alguns resultados relacionados a números de Liouville
}

Elaine Cristine de Souza Silva 
Elaine Cristine de Souza Silva

\title{
Alguns resultados relacionados a números de Liouville
}

\author{
Dissertação apresentada ao Programa de Pós- \\ Graduação em Matemática da Universidade de \\ Brasília, como requisito parcial para obtenção do título \\ de Mestre em Matemática. \\ Orientador: Prof. Dr. Diego Marques Ferreira.
}

Brasília 
Ficha catalográfica elaborada automaticamente, com os dados fornecidos pelo(a) autor(a)

\begin{tabular}{|c|c|}
\hline S586a & $\begin{array}{l}\text { Silva, Elaine Cristine de Souza } \\
\text { Alguns resultados relacionados a números de } \\
\text { Liouville / Elaine Cristine de Souza Silva; } \\
\text { orientador Diego Marques Ferreira. -- Brasília, } 2015 . \\
\quad 70 \mathrm{p} .\end{array}$ \\
\hline & $\begin{array}{l}\text { Dissertação (Mestrado - Mestrado em Matemática) -- } \\
\text { Universidade de Brasília, } 2015 \text {. }\end{array}$ \\
\hline & $\begin{array}{l}\text { 1. Números de Liouville. } 2 \text {. Conjectura de } \\
\text { Schanuel. 3. Conjuntos \$G_ldelta\$. 4. Decomposições. } \\
\text { 5. Funções localmente injetivas. I. Ferreira, Diego } \\
\text { Marques, orient. II. Título. }\end{array}$ \\
\hline
\end{tabular}


Universidade de Brasília

Instituto de Ciências Exatas

Departamento de Matemática

\section{Alguns resultados relacionados a números de Liouville}

por

\section{Elaine Cristine de Souza Silva*}

Dissertação apresentada ao Corpo Docente do Programa de Pós-Graduação em Matemática - UnB, como requisito parcial para obtenção do grau de

\section{MESTRE EM MATEMÁTICA}

Brasília, 11 de março de 2015.

Comissão examinadora:

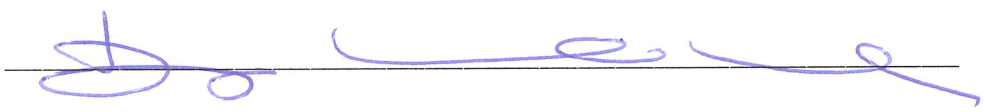

Prof. Dr. Diego Marques Ferreira - UNB (Orientador)

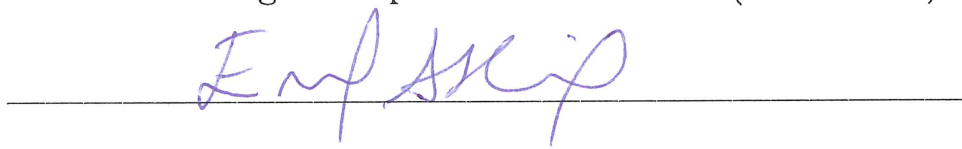

Prof. Dr. Emanuel Augusto de Souza Carneiro - IMPA

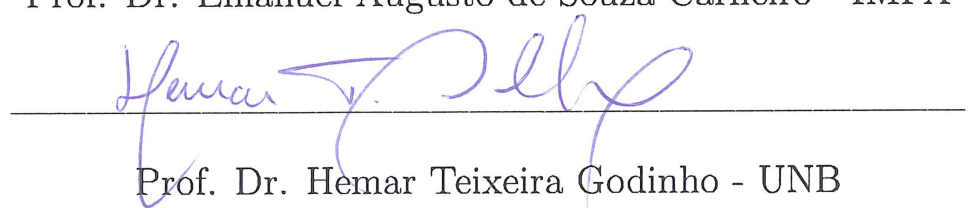

* A autora foi bolsista CAPES e CNPq durante a elaboração desta dissertação. 
À minha mãe e aos meus avós. 


\section{Agradecimentos}

Agradeço a Deus, ser supremo, fonte de luz e inspiração, presente em todos os momentos de minha vida.

Agradeço ao professor Diego Marques pela oportunidade única de trabalhar sob sua orientação, o que me possibilitou uma experiência grandiosa. Agradeço por sua dedicação, paciência e competência profissional.

Agradeço à minha família e aos meus amigos, pelas orações e pelo apoio na luta pelos meus sonhos. Em especial, agradeço à minha mãe, Maria Eliane de Souza Silva, por ser meu grande exemplo de força e determinação; aos meus avós, Antonia de Souza Silva e Francisco Lima da Silva, por nunca me deixarem fraquejar na fé em Deus; ao meu namorado, Carlos Gutierrez, que tem trazido alegria e leveza para os meus dias, pelo carinho, pelas conversas que me confortam e por me incentivar tanto; ao meu padrasto, Junior, à minha sogra, dona Alda, ao meu cunhado, Carlos Williamberg, aos meus padrinhos de batismo, Leidimar e Marcos, e à minha madrinha de crisma, Eliúde, pela atenção a mim dispensada; à Lesse, à Kika e à Locrécia, pela acolhida sempre calorosa; aos meus irmãos, Erika Joyce Silva Lima e Erick Jhone Silva Lima, meus primeiros alunos, que me proporcionaram a oportunidade de conhecer, na infância, a beleza da docência.

Agradeço a todas as pessoas que me deram suporte quanto tive que mudar de cidade para iniciar uma nova jornada. Em especial, agradeço à professora Cristina Fontenele, ao professor José Berto, ao senhor Dario Catunda, à dona Dione, à comunidade Nossa Senhora das Dores, aos professores da minha escola 
de ensino médio, à Organização Barreira Amigos Solidários e à Prefeitura Municipal de Barreira. Sem vocês, tudo teria sido mais complicado.

Agradeço às Escolas Municipais de Educação Infantil e Ensino Fundamental Francisco Ramos de Albuquerque e Francisco Correia Lima e à Escola Estadual de Ensino Médio Danísio Dalton da Rocha Corrêa. Agradeço ainda a todos os professores dessas escolas que me deram a base educacional e, principalmente, a base ética e moral para lutar pelo que acredito de maneira correta.

Agradeço ao Instituto Federal de Educação, Ciência e Tecnologia do Ceará (IFCE) pela oportunidade de cursar Licenciatura em Matemática. Agradeço a todos os professores e alunos dessa instituição que contribuíram para minha formação humana e acadêmica. Em especial, agradeço aos pais e mães que ali obtive: ao professor Aluísio Cabral de Lima, que acreditou em mim e foi o primeiro a me incentivar a fazer mestrado; ao professor Angelo Papa Neto, que me apresentou ao lindo mundo da Teoria dos Números; ao professor Francisco Gevane Muniz Cunha, pela generosidade e compreensão em diversos momentos; às professoras Izaíra Machado Evangelista e Tereza Cristina Valverde de Araújo Alves, que plantaram em mim a semente da pesquisa; ao professor Jânio Kléo de Sousa Castro, pela animação fascinante ao discutir problemas matemáticos e pelas sugestões que tanto contribuíram para a melhoria dessa dissertação; à professora Maria Eugênia Canto Cabral, uma verdadeira inspiração para os meus sonhos; aos professores Breves, Diego Eloi, Dora, Esdras, Lucineide, Luiza, Núbia, Paulo Maia, Simone, Stálio e Valberto, que têm acompanhado minha trajetória durante o mestrado e me recebido com tanta afeição sempre que vou ao IFCE.

Agradeço ao Departamento de Matemática da Universidade de Brasília, por acreditar em mim e me aceitar no seu programa de mestrado. Agradeço aos professores Noraí Romeu Rocco, Ricardo Ruviaro, João Paulo dos Santos, Ary Vasconcelos, Leandro Cioletti, Jiazheng Zhou, Diego Marques e Ricardo Parreira, pelas aulas maravilhosas que tanto contribuíram para a minha formação; à professora Liliane Maia, coordenadora da pós-graduação durante boa parte 
do meu curso, pela sua dedicação; à Bruna, à Claudia e à Eliana, pela eficiência e carisma na execução de seus trabalhos na secretaria de pós-graduação desse departamento.

Agradeço a todos os colegas que iniciaram comigo o mestrado e aqueles que conheci ao decorrer dos semestres, em especial Humberto, do Programa de Pós-Graduação em Linguística, Flor, do Programa de Pós-Graduação em Ciências da Informação, Carol, do Programa de Pós-Graduação em Desenvolvimento Sustentável, Aderson, Alexandre, Christie (Chris), Evelize, Grigório, Hudson, Ilton, Jamer, Katherine, Leandro, Leonardo, Luryane, Michel, Pedro, Ricardo, Wesley, Gabriel, Lumena (Sra. Borges), Rodrigo, Bruno Miranda, Daiane, Filipe, Gérsica, José, Josimar, Lucimeire, Gisele, Marcos e Raimundo, do Programa de Pós-Graduação em Matemática, pelas trocas, pelas conversas e pelo companheirismo. Agradeço ainda ao meu amigo Valter Borges, pela amizade e pelo conhecimento compartilhado, em particular, por me mostrar o teorema que hoje considero ser um dos meus preferidos e que foi uma ferramenta importante para o desenvolvimento deste trabalho.

Agradeço aos professores Diego Marques, Emanuel Carneiro e Hemar Godinho, que compuseram a banca avaliadora. Foi notável a atenção minuciosa que deram ao trabalho fazendo que este se tornasse melhor.

Por fim, agradeço ao CNPQ e à CAPES, pelo apoio financeiro na realização desta pesquisa. 
"Toda ciência, quando não a serviço do poder e da dominação, era para ele 'esta harmonia - mais ou menos vasta e mais ou menos rica conforme a época - que se desfralda no curso das gerações e dos séculos pelo dedicado contraponto de todos os tópicos que vão brotando um depois do outro, como que convocados do Nada '."

João Moreira Salles

( Sobre o matemático Alexander Grothendieck [1928-2014] ) 
"Opte pelo que faz o seu coração vibrar."

Osho

"Não tenhas medo, basta ter fé."

Mc 5,36b 


\section{Resumo}

Esta dissertação trata dos números de Liouville. O estudo foi baseado nos trabalhos de Burger, Caveny, Kumar, Thangadurai e Waldschmidt. Dentre os principais resultados deste trabalho, destacam-se: a generalização de um resultado de Erdös, ao provar que alguns números reais podem ser escritos como $F(\sigma, \tau)$, onde $\sigma$ e $\tau$ são números de Liouville, para uma classe muito grande de funções $F(x, y)$; a determinação de condições suficientes para que a potenciação de números transcendentes seja um número transcendente; e a apresentação de resultados recentes sobre independência algébrica relacionados com os números de Liouville e a Conjectura de Schanuel.

Palavras-chave: Números de Liouville. Conjectura de Schanuel. Conjuntos $G_{\delta}$. Decomposições. Funções localmente injetivas. 


\section{Abstract}

This work is about Liouville numbers. The study was based on works due to Burger, Caveny, Kumar, Thangadurai and Waldschmidt. Among the main results, we highlight: a generalization of an Erdös result, proving that some real numbers can be written as $F(\sigma, \tau)$, where $\sigma$ and $\tau$ are Liouville numbers, for a very large class of functions $F(x, y)$; some sufficient conditions for which the power of two transcendental numbers is still transcendental; and some recent results about algebraic independence related to Liouville numbers and Schanuel's conjecture.

Keywords: Liouville Numbers. Schanuel's Conjecture. $G_{\delta}$-set. Decompositions. Locally injective functions. 


\section{Sumário}

$\begin{array}{ll}\text { Introdução } & 1\end{array}$

1 Números Transcendentes 3

1.1 Números de Liouville . . . . . . . . . . . . . . . . . . . . 4

1.2 Conjectura de Schanuel . . . . . . . . . . . . . . . . . . . 12

1.2.1 Teorema Lindemann-Weierstrass . . . . . . . . . . . . 15

1.2.2 Teorema de Gelfond-Schneider . . . . . . . . . . . . . 16

1.2 .3 Teorema de Baker . . . . . . . . . . . . . . . . 17

1.2.4 Conjectura de Schanuel e as relações entre $e$ e $\pi \ldots$. . 19

2 Números de Liouville e a Conjectura de Schanuel 21

2.1 Preliminares . . . . . . . . . . . . . . . . . . . . . . . . 22

2.2 Lemas auxiliares . . . . . . . . . . . . . . 26

2.3 Prova do teorema . . . . . . . . . . . . . . 37

3 Sobre Decomposições de Liouville 42

3.1 Um teorema de Erdös . . . . . . . . . . . . . . . . . . . . . 43

3.2 Teorema de decomposição . . . . . . . . . . . . . . 46

4 Potenciação de Transcendentes $\quad 51$

5 Números de Liouville e a Propriedade $G_{\delta} \quad \mathbf{5 8}$

5.1 Aplicação da Proposição 5.1 aos números de Liouville . . . . . . 59

5.2 Outros teoremas . . . . . . . . . . . . . . . . . . . . 64 
Referências Bibliográficas 


\section{Introdução}

A Teoria dos Números Transcendentes teve início em maio de 1844, quando o matemático francês Joseph Liouville exibiu os primeiros exemplos de números transcendentes (ver [13]) e provou que, se um número real $\alpha$ é algébrico de grau $n>1$, então existe uma constante $C>0$ tal que $|\alpha-p / q|>C q^{-n}$, para todo $p / q \in \mathbb{Q}, q>1$ (ver [14]). Esse resultado é conhecido como Teorema de Liouville e estabelece um critério para determinar a transcendência de um número real não racional.

Em 1851, Liouville publicou um artigo em que utiliza o fato acima para provar a transcendência dos, agora chamados, números de Liouville: um número real $\xi$ é chamado de número de Liouville se existe uma sequência de racionais distintos $\left(p_{n} / q_{n}\right)_{n \geq 1}$, de modo que $0<\left|\xi-p_{n} / q_{n}\right|<q_{n}^{-n}$. Os primeiros exemplos de números transcendentes, exibidos em 1844, satisfazem essas condições e são, portanto, números de Liouville.

Em 1962, Erdös [7] provou que todo número real pode ser representado como uma soma de dois números de Liouville. Ele apresentou duas provas, uma construtiva (em que os números de Liouville são explicitados) e uma prova não construtiva (em que ele utiliza as propriedades de conjunto $G_{\delta}$ ). Esse resultado é bem interessante, uma vez que o conjunto dos números de Liouville tem medida nula em $\mathbb{R}$.

O resultado de Erdös pode ser reescrito como: para todo $\alpha \in \mathbb{R}$, existem números de Liouville $\sigma$ e $\tau$ tais que $f(\sigma, \tau)=\alpha$, onde $f(x, y)=x+y$. Em 1996, Burger [3] generalizou esse resultado para uma classe mais geral de funções. 
Em particular, o resultado de Burger garante que, dado um número algébrico $\alpha$, sob certas condições, existem números de Liouville $\sigma$ e $\tau$, tais que $\sigma^{\tau}=\alpha$. Em 1993, Caveny [4] já tinha estabelecido condições suficientes para que $\sigma^{\tau}$ fosse transcendente, quando $\sigma$ e $\tau$ são transcendentes.

Em 2014, Kumar, Thangadurai e Waldschmidt [9] provaram diversos resultados sobre o comportamento dos números de Liouville sob a ação de funções contínuas, além de produzirem novos resultados sobre independência algébrica relacionados com os números de Liouville e a Conjectura de Schanuel.

Muitos outros resultados sobre números de Liouville vêm sendo apresentados no decorrer dos anos. Este trabalho tem como proposta mostrar alguns desses resultados, através de uma pesquisa realizada com base nos artigos [3], [4] e [9]. 


\section{Capítulo 1}

\section{Números Transcendentes}

O objetivo principal deste capítulo é apresentar algumas definições e resultados fundamentais em Teoria dos Números Transcendentes, com foco em números de Liouville e na Conjectura de Schanuel.

Definição 1.1 Seja $L \mid K$ uma extensão de corpos. Dizemos que $\alpha \in L$ é algébrico sobre $K$, quando existe $P \in K[x]$, não nulo, tal que $P(\alpha)=0$. Caso contrário, dizemos que a é transcendente sobre $K$.

Quando um número complexo é algébrico sobre $\mathbb{Q}$, dizemos simplesmente que ele é algébrico e denotamos por $\overline{\mathbb{Q}}$ o conjunto desses números, que constitui um corpo (ver [18, p. 70]). É possível provar que um número complexo $\alpha$ é algébrico sobre $\mathbb{Q}$ se, e somente se é algébrico sobre $\overline{\mathbb{Q}}$. Números não algébricos são chamados transcendentes. Denotaremos por $\mathbb{A}$ o conjunto $\overline{\mathbb{Q}} \cap \mathbb{R}$ dos números algébricos reais e por $\mathbb{T}$ o conjunto $\overline{\mathbb{Q}}^{c} \cap \mathbb{R}$ dos números transcendentes reais.

Exemplo 1.2 Todo número racional é algébrico, pois, $\frac{a}{b} \in \mathbb{Q}$ é raiz de $P(x)=$ $b x-a$. Contudo, existem números complexos irracionais que são algébricos, como $\sqrt{2}$ e $i$, que são raízes de $x^{2}-2$ e $x^{2}+1$, respectivamente. 


\subsection{Números de Liouville}

A definição de números transcendentes é do século XVIII e, segundo Euler (1707 - 1783), esses números são chamados transcendentes porque "transcendem" o poder das operações algébricas. Mas foi no século XIX que verificou-se a existência desses números quando, em 13 de maio de 1844, Liouville apresentou, em uma comunicação verbal, os primeiros exemplos de números transcendentes (Ver [13]). Nesse mesmo ano, Liouville apresentou um resultado que determinava condições necessárias para que um número $\alpha \in \mathbb{R} \backslash \mathbb{Q}$ fosse algébrico (Ver [14]). Esse resultado é conhecido como Teorema de Liouville.

Alguns anos depois, Liouville publicou um artigo complementando os resultados anteriores (Ver [15]). Nesse artigo, ele construiu uma classe de números em $\mathbb{R} \backslash \mathbb{Q}$ que não satisfaziam as condições necessárias para serem algébricos (que haviam sido apresentadas em [14]), sendo, portanto, transcendentes. Os números dessa classe são conhecidos como números de Liouville e nela também estão aqueles exibidos em 13 de maio de 1844.

Definição 1.3 Se $\alpha \in \mathbb{C}$ é um número algébrico, definimos o polinômio minimal de a como o polinômio mônico (isto é, coeficiente líder igual a 1) de menor grau, com coeficientes racionais, que tem $\alpha$ como raiz. Nesse caso, o grau de a é definido como o grau do seu polinômio minimal.

Exemplo 1.4 Um número é racional se, e somente se, é algébrico de grau 1. Isto é, se $\alpha$ é algébrico de grau $n \geq 2$, então, $\alpha$ é irracional.

Teorema 1.5 (Teorema de Liouville) Seja $\alpha \in \mathbb{R}$ um número algébrico de grau $n \geq 2$. Então, existe uma constante $A=A(\alpha)>0$ tal que

$$
\left|\alpha-\frac{p}{q}\right|>\frac{A}{q^{n}}
$$

para todo $\frac{p}{q} \in \mathbb{Q}$. 
Demonstração. Ver [18, p.82].

Sabemos que o conjunto dos números racionais é denso em $\mathbb{R}$. Logo, é possível aproximar qualquer número real por números racionais. Contudo, o Teorema de Liouville afirma que números algébricos (reais) não racionais não podem ser muito "bem aproximados" por racionais, no sentido em que qualquer aproximação tem que respeitar esse comportamento. O que Liouville fez depois foi construir números reais não racionais que podem ser muito "bem aproximados" por racionais e, portanto, não são algébricos.

Definição 1.6 Um número real $\xi$ é chamado número de Liouville se existir uma sequência infinita de racionais $\left(\frac{p_{j}}{q_{j}}\right)_{j \geq 1}$ tal que $q_{j}>1 e$

$$
0<\left|\xi-\frac{p_{j}}{q_{j}}\right|<\frac{1}{q_{j}^{j}}
$$

para todo $j \geq 1$. Denotamos por $\mathbb{L}$ o conjuntos dos números de Liouville.

Observação 1.7 Diremos que uma sequência é infinita se possuir uma subsequência de termos distintos.

Apresentaremos alguns resultados que serão utilizados para garantir a transcendência dos números de Liouville.

Proposição 1.8 A sequência $\left(q_{j}\right)_{j \geq 1}$ é ilimitada.

Demonstração. Ver [18, p. 83].

Proposição 1.9 Todo número de Liouville é irracional.

Demonstração. Ver [18, p. 83].

Teorema 1.10 Todo número de Liouville é transcendente. 
Demonstração. Seja $\xi$ um número de Liouville. Vamos supor, por absurdo, que $\xi$ é algébrico. Pela Proposição 1.9, $\xi$ tem grau $n$ maior do que 1. Assim, pelo Teorema de Liouville, existe uma constante $A>0$ tal que, para todo $\frac{p}{q} \in \mathbb{Q}$

$$
\left|\xi-\frac{p}{q}\right|>\frac{A}{q^{n}}
$$

Em particular,

$$
\frac{A}{q_{j}^{n}}<\left|\xi-\frac{p_{j}}{q_{j}}\right|<\frac{1}{q_{j}^{j}}
$$

para todo $j \geq 1$. Em vista disso, $q_{j}^{j-n}<1 / A$. Isso contradiz a Proposição 1.8 .

A seguir, exibimos nosso primeiro exemplo de número de Liouville, conhecido como a constante de Liouville.

Exemplo 1.11 (Constante de Liouville) O número

$$
l=\sum_{n=1}^{\infty} 10^{-n !}
$$

é um número de Liouville. Para provar isso, consideramos as sequências de inteiros

$$
p_{j}=\sum_{n=1}^{j} 10^{j !-n !} \text { e } q_{j}=10^{j !}
$$

Observe que, $\left(\frac{p_{j}}{q_{j}}\right)_{j \geq 1}$ é uma sequência infinita de racionais. Além disso,

$$
\begin{aligned}
\left|l-\frac{p_{j}}{q_{j}}\right| & =\sum_{n=j+1}^{\infty} 10^{-n !}=\sum_{n=1}^{\infty} 10^{-(j+n) !} \\
& =\sum_{n=1}^{\infty} \frac{10^{-(j+1) !}}{10^{(j+n) !-(j+1) !}} \leq \sum_{n=0}^{\infty} \frac{10^{-(j+1) !}}{10^{n}} \\
& =\frac{10}{9 \cdot 10^{(j+1) !}}<\frac{1}{10^{(j+1) !-1}} \leq \frac{1}{10^{j \cdot j !}}=\frac{1}{q_{j}^{j}} .
\end{aligned}
$$


Observação 1.12 Argumentos similares aos vistos no exemplo anterior podem ser utilizados para provar que $\sum_{n=1}^{\infty} a^{-n !}$ é um número de Liouville, para cada inteiro $a \geq 2$.

Em 1906, Maillet [16] provou que a imagem de um número de Liouville por uma função racional não constante é um número de Liouville. A seguir provaremos esse resultado.

Proposição 1.13 Se $f \in \mathbb{Q}(x)$ é uma função racional não constante. Então, $f(\mathbb{L}) \subset \mathbb{L}$.

Demonstração. Sejam $P, Q \in \mathbb{Q}[x]$ tais que $f(x)=P(x) / Q(x)$. Dado $\xi \in \mathbb{L}$, existe $I \subset[\xi-1, \xi+1]$ um intervalo fechado tal que $\xi \in I$ e $Q(x) \cdot f^{\prime}(x) \neq 0$, para cada $x \in I$. Podemos supor que existe uma sequência $\left(\frac{p_{j}}{q_{j}}\right)_{j \geq 1}$ de racionais distintos, $\operatorname{com} p_{j} / q_{j} \in I, q_{j}>1 \mathrm{e}$

$$
\left|\xi-\frac{p_{j}}{q_{j}}\right|<\frac{1}{q_{j}^{j}}
$$

Para cada $j \geq 1$, utilizaremos o Teorema do Valor Médio para o intervalo com extremos $\xi$ e $p_{j} / q_{j}$. Assim, existe $\zeta_{j}$ nesse intervalo tal que

$$
f(\xi)-f\left(\frac{p_{j}}{q_{j}}\right)=f^{\prime}\left(\zeta_{j}\right)\left(\xi-\frac{p_{j}}{q_{j}}\right) .
$$

Pelo Teorema de Weierstrass, existe $\alpha \in I$ tal que $\left|f^{\prime}(\alpha)\right| \geq\left|f^{\prime}(x)\right|$ para todo $x \in I$. Em particular, $\left|f^{\prime}(\alpha)\right| \geq\left|f^{\prime}\left(\zeta_{j}\right)\right|$, para todo $j \in \mathbb{N}$. Portanto,

$$
0<\left|f(\xi)-f\left(\frac{p_{j}}{q_{j}}\right)\right| \leq\left|f^{\prime}(\alpha)\right| \xi-\frac{p_{j}}{q_{j}} \mid<\frac{\left|f^{\prime}(\alpha)\right|}{q_{j}^{j}}
$$

Observe que, se $P(x)=\sum_{i=0}^{n} a_{i} x^{i}$ e $Q(x)=\sum_{i=0}^{m} b_{i} x^{i}$, então,

$$
f\left(\frac{p_{j}}{q_{j}}\right)=\frac{q_{j}^{m}\left(a_{0} q_{j}^{n}+a_{1} p_{j} q_{j}^{n-1}+\ldots+a_{n} p_{j}^{n}\right)}{q_{j}^{n}\left(b_{0} q_{j}^{m}+b_{1} p_{j} q_{j}^{m-1}+\ldots+b_{m} p_{j}^{m}\right)} .
$$


Tome

$$
A_{j}=q_{j}^{m}\left(a_{0} q_{j}^{n}+a_{1} p_{j} q_{j}^{n-1}+\ldots+a_{n} p_{j}^{n}\right)(-1)^{w_{j}}
$$

e

$$
B_{j}=q_{j}^{n}\left(b_{0} q_{j}^{m}+b_{1} p_{j} q_{j}^{m-1}+\ldots+b_{m} p_{j}^{m}\right)(-1)^{w_{j}},
$$

onde

- $w_{j}=0$, se $q_{j}^{n}\left(b_{0} q_{j}^{m}+b_{1} p_{j} q_{j}^{m-1}+\ldots+b_{m} p_{j}^{m}\right) \geq 1$;

- $w_{j}=1$, se $q_{j}^{n}\left(b_{0} q_{j}^{m}+b_{1} p_{j} q_{j}^{m-1}+\ldots+b_{m} p_{j}^{m}\right) \leq-1$.

De $\left|\xi-p_{j} / q_{j}\right|<1$, segue que $\left|p_{j}\right|<(1+|\xi|) q_{j}$. Assim,

$$
\begin{aligned}
\left|B_{j}\right| & =\left|q_{j}^{n}\left(b_{0} q_{j}^{m}+b_{1} p_{j} q_{j}^{m-1}+\ldots+b_{m} p_{j}^{m}\right)\right| \\
& \leq\left|b_{0} q_{j}^{n+m}\right|+\left|b_{1} p_{j} q_{j}^{n+m-1}\right|+\ldots+\left|b_{m} p_{j}^{m} q_{j}^{n}\right| \\
& <\left|b_{0}\right|\left|q_{j}^{n+m}\right|+\left|b_{1}\right|(1+|\xi|)\left|q_{j}^{n+m}\right|+\ldots+\left|b_{m}\right|(1+|\xi|)^{m}\left|q_{j}^{n+m}\right| \\
& \leq L(Q) \theta^{m} q_{j}^{m+n},
\end{aligned}
$$

em que $L(Q)=\left|b_{0}\right|+\left|b_{1}\right|+\ldots+\left|b_{m}\right|$ e $\theta=1+|\xi|$. Segue que $B_{j} \leq L(Q) \theta^{m} q_{j}^{m+n}$.

De (1.1),

$$
0<\left|f(\xi)-f\left(\frac{p_{j}}{q_{j}}\right)\right|<\frac{\left|f^{\prime}(\alpha)\right|}{q_{j}^{j}} \leq \frac{\left|f^{\prime}(\alpha)\right|}{\left(\frac{B_{j}}{L(Q) \theta^{m}}\right)^{\frac{j}{m+n}}}=\frac{\left|f^{\prime}(\alpha)\right|\left(L(Q) \theta^{m}\right)^{\frac{j}{m+n}}}{B_{j}^{\frac{j}{m+n}}} .
$$

Observe que $f\left(p_{j} / q_{j}\right)=A_{j} / B_{j}$ e que $\left(B_{j}\right)_{j \geq 1}$ não pode ser limitada, já que $q_{j}$ é ilimitada e $b_{0} q_{j}^{m}+b_{1} p_{j} q_{j}^{m-1}+\ldots+b_{m} p_{j}^{m} \in \mathbb{Z}^{*}$. Sendo assim,

$$
\lim _{j \longrightarrow \infty} \frac{\left|f^{\prime}(\alpha)\right|\left(L(Q) \theta^{m}\right)^{\frac{j}{m+n}}}{B_{j}^{\frac{j}{2(m+n)}}}=0
$$

e, portanto, existe $C>0$, tal que

$$
\left|\frac{\left|f^{\prime}(\alpha)\right|\left(L(Q) \theta^{m}\right)^{\frac{j}{m+n}}}{B_{j}^{\frac{j}{2(m+n)}}}\right|<C .
$$


Escolhemos $j_{1}$, de modo que $C<B_{j_{1}}^{\frac{j_{1}}{2(m+n)}-1}$ e, para cada $i>1$, escolhemos $j_{i}$, de modo que $j_{i}>j_{i-1}, B_{j_{i}} \notin\left\{B_{j_{1}}, \ldots, B_{j_{n-1}}\right\}$ e $C<B_{j_{i}}^{\frac{j_{i}}{2(m+n)}-i}$.

Por fim, definimos $\frac{c_{i}}{d_{i}}=\frac{A_{j_{i}}}{B_{j_{i}}}$ e obtemos

$$
\begin{array}{r}
0<\left|f(\xi)-\frac{c_{i}}{d_{i}}\right|=\left|f(\xi)-\frac{A_{j_{i}}}{B_{j_{i}}}\right|=\left|f(\xi)-f\left(\frac{p_{j_{i}}}{q_{j_{i}}}\right)\right| \\
<\frac{\left|f^{\prime}(\alpha)\right|\left(L(Q) \theta^{m}\right)^{\frac{j_{i}}{m+n}}}{B_{j_{i}}^{\frac{j_{i}}{m+n}}}<\frac{C}{B_{j_{i}}^{\frac{j_{i}}{2(m+n)}}}<\frac{1}{B_{j_{i}}^{i}}=\frac{1}{d_{i}^{i}} .
\end{array}
$$

Portanto, $f(\xi)$ é um número de Liouville.

Com base nos resultados anteriores, sabemos que todo número de Liouville é transcendente e que existem infinitos números de Liouville, logo, existem infinitos números transcendentes. Em vista disso, surge um questionamento natural:

Todo número transcendente é de Liouville?

Com a finalidade de responder essa pergunta, apresentaremos alguns resultados.

Proposição 1.14 O conjunto dos números algébricos é enumerável.

Demonstração. Ver [18, p. 66].

Observe que, com esse resultado, conseguimos concluir que existem números reais que são transcendentes e que existe uma quantidade não enumerável desses números, caso contrário, o conjunto dos números reais seria enumerável.

É interessante observar que, quando Liouville exibiu os primeiros exemplos de números transcendentes, em 1844, ainda não existia esse conceito de enumerabilidade, uma vez que esse conceito deve-se a Cantor que nasceu em 1845, um ano depois que Liouville exibiu esses números.

A seguir, relembramos a definição de conjuntos de medida (de Lebesgue) nula em $\mathbb{R}$. 
Definição 1.15 Um conjunto $A \subset \mathbb{R}$ tem medida (de Lebesgue) nula, $e$ escrevemos $m(A)=0$ se, para todo $\varepsilon>0$, existe uma quantidade enumerável de intervalos abertos $\left(I_{n}\right)_{n \geq 1}$ tais que $A \subset \bigcup_{n \geq 1} I_{n}$ e $\sum_{n=1}^{\infty}\left|I_{n}\right|<\varepsilon$.

Proposição 1.16 Se $E \subset \mathbb{R}$ é enumerável, então, E tem medida nula.

Demonstração. Ver [18, p. 67].

Dizemos que uma condição é satisfeita por quase todos os números reais, se o subconjunto de $\mathbb{R}$ dos elementos que não satisfazem tal condição tem medida nula.

Proposição 1.17 Quase todo número real é transcendente.

Demonstração. Pela Proposição 1.14, segue que o conjunto dos números algébricos é enumerável, isto é, $\mathbb{A}$ é enumerável. Segue, da Proposição 1.16, que $\mathbb{A}$ tem medida nula. Com isso concluímos que quase todo número real é transcendente.

A seguir, provaremos uma equivalência para a definição de número de Liouville.

Lema 1.18 छ é um número de Liouville se, e somente se, para todo $n \geq 1$, existe $\frac{p}{q} \in \mathbb{Q}$, tal que $q>1$ e

$$
0<\left|\xi-\frac{p}{q}\right|<\frac{1}{q^{n}}
$$

Demonstração. Se $\xi$ é um número de Liouville, dado $n \in \mathbb{N}$, podemos tomar $p=p_{n}$ e $q=q_{n}$. Reciprocamente, dado $n \in \mathbb{N}$, vamos escolher $\frac{p_{n}}{q_{n}} \in \mathbb{Q}$ de modo que $q_{n}>1$ e

$$
0<\left|\xi-\frac{p_{n}}{q_{n}}\right|<\frac{1}{q_{n}^{n}}
$$


Seja

$$
A=\bigcup_{n \geq 1}\left\{\frac{p_{n}}{q_{n}}\right\}
$$

Se $A$ for finito, então existe $\frac{p}{q} \in A$ tal que $|\xi-p / q|<q^{-n}$ para $n \in \mathbb{N}^{\prime}$, com $\mathbb{N}^{\prime} \subset \mathbb{N}$ infinito. Assim, $\xi=\frac{p}{q}$, contradizendo $|\xi-p / q|>0$. Portanto, $A$ é infinito. Concluímos que $\xi$ é um número de Liouville.

Teorema 1.19 O conjunto dos números de Liouville tem medida nula em $\mathbb{R}$.

Demonstração. É suficiente provar que $\mathbb{L} \cap[k, k+1]$ tem medida nula, para cada $k \in \mathbb{Z}$. Mostraremos que $\mathbb{L} \cap[0,1]$ tem medida nula, pois os outros casos seguem de modo análogo. Seja $\varepsilon>0$.

AFIRMAÇÃO 1: Existe $n \in \mathbb{N}$ tal que $\sum_{b=2}^{\infty} \frac{4}{b^{n-1}}<\varepsilon$.

De fato, se $a_{k}=\sum_{b=2}^{\infty} \frac{4}{b^{k-1}}$, com $k \geq 3$, temos

$$
0<a_{k}=\sum_{b=2}^{\infty} \frac{4}{b^{k-3} b^{2}} \leq \frac{4}{2^{k-3}} \sum_{b=2}^{\infty} \frac{1}{b^{2}}=\frac{4}{2^{k-3}} \cdot\left(\frac{\pi^{2}}{6}-1\right)
$$

uma vez que $\sum_{b=1}^{\infty} \frac{1}{b^{2}}=\frac{\pi^{2}}{6}\left(\right.$ ver [2]). Pelo Teorema do Confronto, $\lim _{k \longrightarrow \infty} a_{k}=0$ e a Afirmação 1 está provada.

Se $\xi \in[0,1] \cap \mathbb{L}$, então, pelo Lema 1.18 , existe $\frac{a}{b} \in \mathbb{Q}$, com $b>1$, tal que

$$
\left|\xi-\frac{a}{b}\right|<\frac{1}{b^{n}} \leq \frac{1}{2^{n}} \leq \frac{1}{2}
$$

para o $n$ da afirmação anterior. Segue que, $a \in[-b / 2,3 b / 2]$.

Como o comprimento desse intervalo é $2 b$ e $a \in \mathbb{Z}$, então, há, no máximo, $2 b$ valores possíveis para $a$, satisfazendo (1.2). Chamaremos $C_{b}$ o conjunto de tais valores. Portanto,

$$
\xi \in \bigcup_{a \in C_{b}}\left(\frac{a}{b}-\frac{1}{b^{n}}, \frac{a}{b}+\frac{1}{b^{n}}\right)
$$


para algum $b \geq 2$. E assim,

$$
\mathbb{L} \cap[0,1] \subset \bigcup_{b \geq 2} \bigcup_{a \in C_{b}}\left(\frac{a}{b}-\frac{1}{b^{n}}, \frac{a}{b}+\frac{1}{b^{n}}\right) .
$$

Por fim, observe que o comprimento do intervalo $\left(a / b-1 / b^{n}, a / b+1 / b^{n}\right)$ é $2 / b^{n}$. Logo,

$$
\sum_{b \geq 2} \sum_{a \in C_{b}} \frac{2}{b^{n}}=\sum_{b \geq 2} \frac{2 b \cdot 2}{b^{n}}=\sum_{b \geq 2} \frac{4}{b^{n-1}}<\varepsilon .
$$

Com esse último resultado, vemos que, se todo número transcendente fosse de Liouville, teríamos $\mathbb{A} \cup \mathbb{L}=\mathbb{R}$ e, assim, o conjunto dos números reais teria medida nula, o que é uma contradição.

Os números e e $\pi$ são exemplos de números transcendentes que não são de Liouville (ver [21, p.330]). Na verdade, com o resultado anterior, concluímos que quase todo número é transcendente, mas quase nenhum é de Liouville. Isso significa que o conjunto dos números de Liouville é pequeno em $\mathbb{R}$, no ponto de vista da Teoria da Medida.

Na próxima seção, falaremos sobre a Conjectura de Schanuel e sua relevância para Teoria dos Números Transcendentes, além de ver alguns exemplos de números transcendentes.

\subsection{Conjectura de Schanuel}

Inicialmente, apresentaremos algumas definições importantes para esta seção e para os capítulos que seguem.

Definição 1.20 Seja $L \mid K$ uma extensão de corpos. Dizemos que $L \mid K$ é uma extensão algébrica se todo $\alpha \in L$ é algébrico sobre $K$. Caso contrário, dizemos que $L \mid K$ é uma extensão transcendente.

Exemplo 1.21 A extensão $\mathbb{Q}(\sqrt{2}) \mid \mathbb{Q}$ é algébrica, enquanto a extensão $\mathbb{Q}(l) \mid \mathbb{Q}$ é transcendente, onde l é a constante de Liouville. 
Definição 1.22 Seja $L \mid K$ uma extensão de corpos. Dizemos que $\alpha_{1}, \ldots, \alpha_{n} \in$ $L$ são algebricamente dependentes sobre $K$ se existir polinômio não constante

$$
P\left(x_{1}, \ldots, x_{n}\right) \in K\left[x_{1}, \ldots, x_{n}\right]
$$

tal que $P\left(\alpha_{1}, \ldots, \alpha_{n}\right)=0$. Caso contrário, dizemos que $\alpha_{1}, \ldots, \alpha_{n} \in L$ são algebricamente independentes sobre $K$.

Se $\alpha_{1}, \ldots, \alpha_{n}$ são números complexos algebricamente dependentes sobre $\mathbb{Q}$ (resp. algebricamente independentes sobre $\mathbb{Q}$ ), dizemos simplesmente que eles são algebricamente dependentes (resp. algebricamente independentes).

Observação 1.23 Note que se $\alpha_{1}, \ldots, \alpha_{n}$ são algebricamente independentes, então, $\alpha_{1}, \ldots, \alpha_{n}$ são todos transcendentes. Entretanto, a recíproca não é verdadeira, por exemplo, os números $l$ e $l^{2}$ são transcendentes, mas são algebricamente dependentes, basta tomar $P(x, y)=x^{2}-y$. Conjectura-se que e e $\pi$ são algebricamente independentes, porém até a transcendência de $e+\pi$ ainda é um problema em aberto.

Definição 1.24 Seja $L \mid K$ uma extensão de corpos. Dizemos que um subconjunto infinito de $L$ é algebricamente independente sobre $K$, se todo subconjunto finito o for.

Observação 1.25 É possível mostrar que $\alpha_{1}, \ldots, \alpha_{n}, \ldots \in L$ são algebricamente dependentes sobre $K$ se, e somente se, existe $n \in \mathbb{N}$, tal que $\alpha_{1}, \ldots, \alpha_{n}$ são algebricamente dependentes sobre $K$.

Definição 1.26 Seja $L \mid K$ uma extensão de corpos. Um conjunto $\mathcal{B} \subset L$ é chamado base de transcendência de $L \mid K$, se $\mathcal{B}$ é algebricamente independente sobre $K$ e $L \mid K(\mathcal{B})$ é uma extensão algébrica.

Exemplo $1.27 O$ conjunto $\mathcal{B}_{1}=\varnothing$ é uma base de transcendência para a extensão $\mathbb{Q}(\sqrt{2}) \mid \mathbb{Q}$ e o conjunto $\mathcal{B}_{2}=\{l\}$ é uma base de transcendência para a extensão $\mathbb{Q}(l) \mid \mathbb{Q}$. 
É possível provar que quaisquer duas bases de transcendência de uma extensão têm a mesma cardinalidade (Ver [23, p. 99]), sendo assim, faz sentido definir grau de transcendência como segue.

Definição 1.28 Seja $L \mid K$ uma extensão de corpos. Definimos o grau de transcendência dessa extensão como a cardinalidade de uma base de transcendência.

Denotamos o grau de transcendência dessa extensão por $\operatorname{grtr}_{K} L$ ou $\operatorname{grtr}(L \mid K)$.

Proposição 1.29 Seja $\mathbb{Q}\left(x_{1}, \ldots, x_{n}\right) \mid \mathbb{Q}$ uma extensão de corpos. É possível obter uma base de transcendência $\mathcal{B}$ contida em $\left\{x_{1}, \ldots, x_{n}\right\}$.

Demonstração. Provaremos o resultado por indução. Seja $n=1$. Se $x_{1} \in \overline{\mathbb{Q}}$, temos $\mathcal{B}=\varnothing$. Se $x_{1} \notin \overline{\mathbb{Q}}$, podemos tomar $\mathcal{B}=\left\{x_{1}\right\}$. Em qualquer caso, $\mathcal{B} \subset\left\{x_{1}\right\}$.

Seja $n>1$. Vamos supor que o resultado é válido para $n-1$. Seja $\mathcal{B}^{\prime} \subset\left\{x_{1}, \ldots, x_{n-1}\right\}$ uma base de transcendência para a extensão

$$
\mathbb{Q}\left(x_{1}, \ldots, x_{n-1}\right) \mid \mathbb{Q} .
$$

Se $x_{n}$ é algébrico sobre $\mathbb{Q}\left(x_{1}, \ldots, x_{n-1}\right)$, podemos tomar $\mathcal{B}=\mathcal{B}^{\prime}$. Se $x_{n}$ é transcendente sobre $\mathbb{Q}\left(x_{1}, \ldots, x_{n-1}\right)$, podemos tomar $\mathcal{B}=\mathcal{B}^{\prime} \cup\left\{x_{n}\right\}$. Em qualquer caso, $\mathcal{B} \subset\left\{x_{1}, \ldots, x_{n}\right\}$.

Através da proposição anterior, concluímos que $\operatorname{grtr}\left(\mathbb{Q}\left(x_{1}, \ldots, x_{n}\right) \mid \mathbb{Q}\right) \leq$ $n$. Em particular, conseguimos um limitante superior para o grau de transcendência da extensão $\mathbb{Q}\left(x_{1}, \ldots, x_{n}, e^{x_{1}}, \ldots, e^{x_{n}}\right) \mid \mathbb{Q}$. A seguir, apresentamos a Conjectura de Schanuel que, se provada, garante um limitante inferior para o grau de transcendência dessa extensão no caso em que $x_{1}, \ldots, x_{n}$ são linearmente independentes.

Conjectura 1.30 (Schanuel) $S e x_{1}, \ldots, x_{n} \in \mathbb{C}$ são linearmente independentes sobre $\mathbb{Q}$, então 


$$
\operatorname{grtr}\left(\mathbb{Q}\left(x_{1}, \ldots, x_{n}, e^{x_{1}}, \ldots, e^{x_{n}}\right) \mid \mathbb{Q}\right) \geq n
$$

A seguir, apresentamos alguns teoremas importantes em Teoria dos Números Transcendentes, que foram provados independentemente da Conjectura de Schanuel (com demonstrações não triviais) e mostraremos que, se comprovada sua veracidade, a Conjectura de Schanuel pode ser utilizada para provar esses teoremas de maneira simples.

\subsubsection{Teorema Lindemann-Weierstrass}

Teorema 1.31 (Lindemann-Weierstrass) Sejam $\alpha_{1}, \ldots, \alpha_{n}$ números algébricos linearmente independentes sobre $\mathbb{Q}$, então $e^{\alpha_{1}}, \ldots, e^{\alpha_{n}}$ são algebricamente independentes.

Demonstração. Ver $[8$, p. 88]

Esse teorema implica a veracidade da Conjectura de Schanuel quando $\alpha_{1}, \ldots, \alpha_{n}$ são números algébricos linearmente independentes. Além disso, se a Conjectura de Schanuel é verdadeira, temos

$$
\operatorname{grtr}\left(\mathbb{Q}\left(\alpha_{1}, \ldots, \alpha_{n}, e^{\alpha_{1}}, \ldots, e^{\alpha_{n}}\right) \mid \mathbb{Q}\right) \geq n
$$

e, como $\alpha_{1}, \ldots, \alpha_{n}$ são algébricos, temos

$$
\operatorname{grtr}\left(\mathbb{Q}\left(e^{\alpha_{1}}, \ldots, e^{\alpha_{n}}\right) \mid \mathbb{Q}\right)=\operatorname{grtr}\left(\mathbb{Q}\left(\alpha_{1}, \ldots, \alpha_{n}, e^{\alpha_{1}}, \ldots, e^{\alpha_{n}}\right) \mid \mathbb{Q}\right) .
$$

Além disso, sabemos que

$$
\operatorname{grtr}\left(\mathbb{Q}\left(e^{\alpha_{1}}, \ldots, e^{\alpha_{n}}\right) \mid \mathbb{Q}\right) \leq n
$$

Por, (1.3), (1.4) e (1.5), obtemos

$$
\operatorname{grtr}\left(\mathbb{Q}\left(e^{\alpha_{1}}, \ldots, e^{\alpha_{n}}\right) \mid \mathbb{Q}\right)=n .
$$

Logo, $e^{\alpha_{1}}, \ldots, e^{\alpha_{n}}$ são algebricamente independentes e, portanto, o Teorema de Lindemann-Weierstrass segue como consequência da Conjectura de Schanuel. 
Exemplo 1.32 Dado a algébrico não nulo, temos que $\{\alpha\}$ é um conjunto linearmente independente sobre $\mathbb{Q}$, segue do Teorema de Lindemann-Weierstrass que $e^{\alpha}$ é transcendente. Daí segue que $\pi$ é transcendente, pois se $\pi$ fosse algébrico, in também seria e $e^{i \pi}=-1$ seria transcendente.

\subsubsection{Teorema de Gelfond-Schneider}

Teorema 1.33 (Gelfond-Schneider) Seja $\alpha \in \overline{\mathbb{Q}} \backslash\{0\}$ e $\beta \in \overline{\mathbb{Q}} \backslash \mathbb{Q}$. Então $\alpha^{\beta}$ é transcendente.

Demonstração. Ver [19, 60].

Observação 1.34 Sejam $\alpha, \beta \in \mathbb{C}$, com $\alpha \neq 0$. Definimos

$$
\alpha^{\beta}:=e^{\beta \log (\alpha)}
$$

O fato de que a função exponencial complexa log está envolvida nessa definição implica que devemos escolher um ramo para que a função exponencial $\alpha^{\beta}$ seja bem definida.

Exemplo 1.35 (Constante de Gelfond) Segue, do Teorema de GelfondSchneider, a transcendência de $e^{\pi}$, pois se $e^{\pi}$ fosse algébrico, $\left(e^{\pi}\right)^{i}=-1$ seria transcendente.

Proposição 1.36 Suponha que a Conjectura de Schanuel é verdadeira. Se $\alpha \in \overline{\mathbb{Q}} \backslash\{0,1\}$ e $\beta \in \overline{\mathbb{Q}} \backslash \mathbb{Q}$, então $\alpha^{\beta}$ e $\log \alpha$ são algebricamente independentes.

Demonstração. Primeiramente, mostraremos que $\beta \log \alpha$ e $\log \alpha$ são linearmente independentes sobre $\mathbb{Q}$, em seguida, aplicaremos a Conjectura de Schanuel.

Sejam $a_{1}$ e $a_{2}$ inteiros tais que $a_{1} \beta \log \alpha+a_{2} \log \alpha=0$. Como, $\alpha \neq 1$, temos $\log \alpha \neq 0$ e assim, $a_{1} \beta+a_{2}=0$. Se $a_{1} \neq 0$, temos $\beta \in \mathbb{Q}$, que é uma contradição. Logo, $a_{1}=0$ e, consequentemente, $a_{2}=0$. 
Se a Conjectura de Schanuel é verdadeira, temos

$$
\operatorname{grtr}\left(\mathbb{Q}\left(\beta \log \alpha, \log \alpha, \alpha^{\beta}, \alpha\right) \mid \mathbb{Q}\right) \geq 2
$$

Além disso, desde que $\alpha, \beta \in \overline{\mathbb{Q}}$,

$$
\operatorname{grtr}\left(\mathbb{Q}\left(\alpha^{\beta}, \log \alpha\right) \mid \mathbb{Q}\right)=\operatorname{grtr}\left(\mathbb{Q}\left(\beta \log \alpha, \log \alpha, \alpha^{\beta}, \alpha\right) \mid \mathbb{Q}\right)
$$

$\mathrm{e}$

$$
\operatorname{grtr}\left(\mathbb{Q}\left(\alpha^{\beta}, \log \alpha\right) \mid \mathbb{Q}\right) \leq 2
$$

Por (1.6), (1.7) e (1.8), temos

$$
\operatorname{grtr}\left(\mathbb{Q}\left(\alpha^{\beta}, \log \alpha\right) \mid \mathbb{Q}\right)=2
$$

Portanto, $\alpha^{\beta}$ e $\log \alpha$ são algebricamente independentes, logo transcendentes.

A partir da Proposição 1.36 e da Observação 1.23, temos que a Conjectura de Schanuel implica a transcendência de $\alpha^{\beta}$ e $\log \alpha$, quando $\alpha \in \overline{\mathbb{Q}} \backslash\{0,1\}$ e $\beta \in \overline{\mathbb{Q}} \backslash \mathbb{Q}$. Em particular, o Teorema de Gelfond-Schneider segue como consequência da Conjectura de Schanuel.

\subsubsection{Teorema de Baker}

O Teorema a seguir foi provado por Alan Baker em 1966. Em reconhecimento de suas contribuições, Baker foi premiado com a medalha Fields em 1970.

Teorema 1.37 (Baker) Sejam $\alpha_{1}, \ldots, \alpha_{n}$ números algébricos não nulos de modo que $\log \alpha_{1}, \ldots, \log \alpha_{n}$ são linearmente independentes sobre $\mathbb{Q}$. Então, $1, \log \alpha_{1}, \ldots, \log \alpha_{n}$ são linearmente independentes sobre $\overline{\mathbb{Q}}$.

Demonstração. Ver [19, p. 84]

Uma consequência interessante do Teorema de Baker é o seguinte resultado, que generaliza o Teorema de Gelfond-Schneider. 
Proposição 1.38 O número $\alpha_{1}^{\beta_{1}} \cdots \alpha_{n}^{\beta_{n}}$ é transcendente para todos os algébricos $\alpha_{1}, \ldots, \alpha_{n}$, diferentes de 0 e 1 , e todos os números algébricos $\beta_{1}, \ldots, \beta_{n}$ com $1, \beta_{1}, \ldots, \beta_{n}$ linearmente independentes sobre $\mathbb{Q}$.

Demonstração. Ver [18, p. 129].

Proposição 1.39 Suponha que a Conjectura de Schanuel é verdadeira. Se $\alpha_{1}, \ldots, \alpha_{n}$ são números algébricos não nulos tais que $\log \alpha_{1}, \ldots, \log \alpha_{n}$ são linearmente independentes sobre $\mathbb{Q}$. Então, $\log \alpha_{1}, \ldots, \log \alpha_{n}$ são algebricamente independentes.

Demonstração. Tome $x_{i}=\log \alpha_{i}$ na Conjectura de Schanuel. Assim,

$$
\begin{gathered}
\operatorname{grtr}\left(\mathbb{Q}\left(\log \alpha_{1}, \ldots, \log \alpha_{n}, \alpha_{1}, \ldots, \alpha_{n}\right) \mid \mathbb{Q}\right) \geq n \\
\operatorname{grtr}\left(\mathbb{Q}\left(\log \alpha_{1}, \ldots, \log \alpha_{n}\right) \mid \mathbb{Q}\right)=\operatorname{grtr}\left(\mathbb{Q}\left(\log \alpha_{1}, \ldots, \log \alpha_{n}, \alpha_{1}, \ldots, \alpha_{n}\right) \mid \mathbb{Q}\right)
\end{gathered}
$$

$\mathrm{e}$

$$
\operatorname{grtr}\left(\mathbb{Q}\left(\log \alpha_{1}, \ldots, \log \alpha_{n}\right) \mid \mathbb{Q}\right) \leq n
$$

Portanto,

$$
\operatorname{grtr}\left(\mathbb{Q}\left(\log \alpha_{1}, \ldots, \log \alpha_{n}\right) \mid \mathbb{Q}\right)=n
$$

Consequentemente, $\log \alpha_{1}, \ldots, \log \alpha_{n}$ são algebricamente independentes.

Observe que, dados $\alpha_{1}, \ldots, \alpha_{n}$ números algébricos não nulos de modo que $\log \alpha_{1}, \ldots, \log \alpha_{n}$ são linearmente independentes sobre $\mathbb{Q}$, a proposição anterior garante que a veracidade da Conjectura de Schanuel implica não só na independência linear de $1, \log \alpha_{1}, \ldots, \log \alpha_{n}$, (ou seja, o Teorema de Baker) como a independência algébrica de $\log \alpha_{1}, \ldots, \log \alpha_{n}$. 


\subsubsection{Conjectura de Schanuel e as relações entre $e$ e $\pi$}

A veracidade da Conjectura de Schanuel tem, como consequência, a resolução de vários problemas em aberto. Dentre eles, a independência algébrica de $e$ e $\pi$.

Teorema 1.40 Se a Conjectura de Schanuel é verdadeira, então e e $\pi$ são algebricamente independentes.

Demonstração. Observe que $i \pi$ e 1 são linearmente independentes sobre $\mathbb{Q}$. Assim, pela Conjectura de Schanuel,

$$
\operatorname{grtr}(\mathbb{Q}(i \pi, 1,-1, e) \mid \mathbb{Q}) \geq 2
$$

Além disso,

$$
\operatorname{grtr}(\mathbb{Q}(i \pi, e) \mid \mathbb{Q})=\operatorname{grtr}(\mathbb{Q}(i \pi, 1,-1, e) \mid \mathbb{Q})
$$

$\mathrm{e}$

$$
\operatorname{grtr}(\mathbb{Q}(i \pi, e) \mid \mathbb{Q}) \leq 2
$$

Portanto,

$$
\operatorname{grtr}(\mathbb{Q}(i \pi, e) \mid \mathbb{Q})=2 .
$$

Logo, $i \pi$ e $e$ são algebricamente independentes, consequentemente, $\pi$ e $e$ são algebricamente independentes.

Essa conjectura tão importante tem muitas outras consequências interessantes, para ver mais algumas, recomendamos [6]. Nesse artigo, os autores mostram que a Conjectura de Schanuel implica um resultado ainda mais forte do que a independência algébrica de $e$ e $\pi$. Eles provaram que a Conjectura de Schanuel implica que $\pi \notin E$, de modo que $E=\bigcup_{n \geq 0} E_{n}$, onde $E_{n}$ é definido indutivamente por 
- $E_{0}=\overline{\mathbb{Q}}$;

- $E_{n}=\overline{E_{n-1}\left(\left\{e^{x}: x \in E_{n-1}\right\}\right)}$, para $n \geq 1$. Em que $\overline{E_{n-1}\left(\left\{e^{x}: x \in E_{n-1}\right\}\right)}$ denota o fecho algébrico de $E_{n-1}\left(\left\{e^{x}: x \in E_{n-1}\right\}\right)$.

Nesta seção, apresentamos a Conjectura de Schanuel e vimos sua relação com alguns teoremas importantes. No próximo capítulo, apresentaremos resultados recentes de independência algébrica relacionados com os números de Liouville e a Conjectura de Schanuel. 


\section{Capítulo 2}

\section{Números de Liouville e a \\ Conjectura de Schanuel}

A Conjectura de Schanuel é, sem dúvida, um dos principais problemas em aberto em Teoria dos Números Transcendentes. Em 2014, Kumar, Thangadurai e Waldschmidt, publicaram o artigo Liouville Numbers and Schanuel's Conjecture. O principal resultado apresentado nesse artigo garante que, para cada par de inteiros positivos $(n, m)$, com $n \geq m \geq 1$, existe uma quantidade não enumerável de $n$-uplas $\left(\xi_{1}, \ldots, \xi_{n}\right)$ consistindo de números reais linearmente independentes sobre $\mathbb{Q}$ tais que os números

$$
\xi_{1}, \ldots, \xi_{n}, e^{\xi_{1}}, \ldots, e^{\xi_{n}}
$$

são todos números de Liouville e o grau de transcendência da extensão

$$
\mathbb{Q}\left(\xi_{1}, \ldots, \xi_{n}, e^{\xi_{1}}, \ldots, e^{\xi_{n}}\right) \mid \mathbb{Q}
$$

é exatamente $n+m$.

Observe que, fixado $n$ tão grande quanto se deseje e tomando $m=n$, esse resultado garante que existe uma quantidade não enumerável de uplas $\xi_{1}, \ldots, \xi_{n}$ tais que

$$
\xi_{1}, \ldots, \xi_{n}, e^{\xi_{1}}, \ldots, e^{\xi_{n}}
$$


são todos números de Liouville algebricamente independentes sobre $\mathbb{Q}$.

Além disso, com o que chamaremos de Teorema de Kumar-ThangaduraiWaldschmidt, conseguimos garantir que, fixado $n$, há uma quantidade nãoenumerável de $n$-uplas $\left(x_{1}, \ldots, x_{n}\right) \in \mathbb{L}^{n}$, com $x_{1}, \ldots x_{n}$ linearmente independentes sobre $\mathbb{Q}$, para os quais

$$
\operatorname{grtr}\left(\mathbb{Q}\left(x_{1}, \ldots, x_{n}, e^{x_{1}}, \ldots, e^{x_{n}}\right) \mid \mathbb{Q}\right) \geq n+1
$$

e assim, para essas $n$-uplas, a Conjectura de Schanuel é verdadeira.

Ainda com respeito a essa conjectura, a partir dessas observações, surge uma pergunta interessante: Se acrescentamos a hipótese de que

$$
x_{1}, \ldots, x_{n}, e^{x_{1}}, \ldots, e^{x_{n}}
$$

são números de Liouville, podemos garantir

$$
\operatorname{grtr}_{\mathbb{Q}} \mathbb{Q}\left(x_{1}, \ldots, x_{n}, e^{x_{1}}, \ldots, e^{x_{n}}\right) \geq n+1 ?
$$

É claro que, com o resultado apresentado, conseguimos garantir apenas para uma quantidade não enumerável, mas não para quaisquer números de Liouville satisfazendo essas condições. De qualquer forma, é um questionamento interessante no que se refere à relação entre os primeiros números transcendentes e a Conjectura de Schanuel.

\subsection{Preliminares}

Nesta seção apresentaremos resultados preliminares que serão usados no decorrer do capítulo.

Observação 2.1 No capítulo anterior, denotamos por $\overline{\mathbb{Q}}$ o conjunto dos números algébricos. Entretanto, neste capítulo, denotaremos por $\bar{A}$ o fecho topológico de $A$ em $X$, dados $X$ um espaço topológico e $A \subset X$. 
Proposição 2.2 Sejam $X$ um espaço topológico e $A_{\alpha} \subset X$, para cada $\alpha \in \Gamma$. Então,

$$
\bigcup_{\alpha \in \Gamma} \overline{A_{\alpha}} \subset \overline{\bigcup_{\alpha \in \Gamma} A_{\alpha}}
$$

Demonstração. Seja

$$
x \in \bigcup_{\alpha \in \Gamma} \overline{A_{\alpha}}
$$

assim, $x \in \overline{A_{\alpha}}$, para algum $\alpha \in \Gamma$. Se $V \subset X$ é um aberto tal que $x \in V$, então, $V \cap A_{\alpha} \neq \varnothing$. Logo,

$$
V \cap \bigcup_{\alpha \in \Gamma} A_{\alpha}=\bigcup_{\alpha \in \Gamma}\left(V \cap A_{\alpha}\right) \neq \varnothing
$$

Concluímos que

$$
x \in \overline{\bigcup_{\alpha \in \Gamma} A_{\alpha}}
$$

Proposição 2.3 Se $A \subset \mathbb{R}$ é um conjunto não enumerável, então $A$ tem ponto de acumulação.

Demonstração. Seja $A \subset \mathbb{R}$ um conjunto não enumerável.

AFIRMAÇÃO 1: Existe $k \in \mathbb{Z}$ tal que $A \cap[k, k+1]$ é infinito.

Sabemos que,

$$
\mathbb{R}=\bigcup_{k \in \mathbb{Z}}[k, k+1]
$$

assim, se $[k, k+1] \cap A$ é finito, para todo $k \in \mathbb{Z}$, então,

$$
A=A \cap \mathbb{R}=A \cap\left(\bigcup_{k \in \mathbb{Z}}[k, k+1]\right)=\bigcup_{k \in \mathbb{Z}}(A \cap[k, k+1])
$$


é uma união enumerável de conjuntos finitos, logo enumerável, o que contradiz a não enumerabilidade de $A$. Portanto, a Afirmação 1 está provada.

Seja $k \in \mathbb{Z}$ tal que $A \cap[k, k+1]$ é infinito e seja $\left(x_{n}\right)_{n \in \mathbb{N}}$ uma sequência de pontos distintos em $A \cap[k, k+1]$. Logo, $\left(x_{n}\right)_{n \in \mathbb{N}}$ é limitada e, pelo Teorema de Bolzano-Weierstrass, $\left(x_{n}\right)_{n \in \mathbb{N}}$ possui subsequência convergente, portanto, $A \cap[k, k+1]$ tem ponto de acumulação e, consequentemente, $A$ tem ponto de acumulação.

Proposição 2.4 Sejam $A: \mathbb{R}_{+} \longrightarrow \mathbb{R}$ e $B: \mathbb{R}_{+} \longrightarrow \mathbb{R}$ funções tais que

$$
\lim _{x \rightarrow \infty} A(x)=+\infty e \lim _{x \rightarrow \infty} \frac{B(x)}{A(x)}=0,
$$

então,

$$
\lim _{x \longrightarrow \infty}(A(x)-B(x))=+\infty
$$

Demonstração. Se $A: \mathbb{R}_{+} \longrightarrow \mathbb{R}$ e $B: \mathbb{R}_{+} \longrightarrow \mathbb{R}$ são funções satisfazendo as hipóteses acima, existe $\delta>0$ (suficientemente grande) tal que $x>\delta$ implica

$$
A(x)>0 \text { e }\left|\frac{B(x)}{A(x)}\right|<\frac{1}{2} .
$$

Assim,

$$
-\frac{A(x)}{2}<B(x)<\frac{A(x)}{2}
$$

e, somando $A(x)$, temos

$$
\frac{A(x)}{2}<A(x)-B(x)<A(x)+\frac{A(x)}{2} .
$$

Como $\lim _{x \longrightarrow \infty} \frac{A(x)}{2}=\infty$, então

$$
\lim _{x \longrightarrow \infty}(A(x)-B(x))=\infty .
$$




\section{TEOREMA DE BAIRE}

Definição 2.5 Seja $X$ um espaço topológico. Dizemos que $A \subset X$ é um conjunto magro em $\boldsymbol{X}$ se $A$ é uma reunião enumerável de conjuntos fechados com interior vazio.

A noção de conjuntos magros em Topologia desempenha, em um certo sentido, papel semelhante ao dos conjuntos de medida nula em Análise.

Exemplo 2.6 O conjunto $\mathbb{Q}$ dos números racionais é conjunto magro em $\mathbb{R}$, pois, sendo $\mathbb{Q}$ enumerável, temos

$$
\mathbb{Q}=\bigcup_{x \in \mathbb{Q}}\{x\}
$$

$e\{x\}$ é um conjunto fechado com interior vazio, para cada $x \in \mathbb{Q}$.

É razoável pensar que todo conjunto magro tem interior vazio, uma vez que é uma reunião enumerável de conjuntos fechados com interior vazio, mas isso não é verdade. Por exemplo, se pensarmos em $\mathbb{Q}$ com a topologia induzida de $\mathbb{R}$, e $\mathbb{Q}_{+}$como um subconjunto de $\mathbb{Q}$, vemos que $\mathbb{Q}_{+}$é um conjunto magro, mas não tem interior vazio em $\mathbb{Q}$.

Definição 2.7 Um espaço topológico no qual todo conjunto magro tem interior vazio é chamado de espaço de Baire.

Teorema 2.8 (Teorema de Baire) Todo espaço métrico completo com a topologia induzida pela métrica é um espaço de Baire.

Sabemos que $\mathbb{R}$ é um espaço métrico completo, logo é um espaço de Baire. Observe ainda que, se $I \subset \mathbb{R}$ é um intervalo fechado com interior não vazio, temos que $I$ é um espaço métrico completo e, em vista disso, $I$ é um espaço de Baire.

Proposição 2.9 Seja $X$ um espaço de Baire, então, todo aberto $A \subset X$ é um espaço de Baire com a topologia induzida. 
Dessa proposição segue que, se $I$ é um intervalo aberto limitado com interior não vazio, ele também é um espaço de Baire. Observe que não poderíamos utilizar apenas o Teorema 2.8 para garantir isso, pois, nesse caso, I não é espaço métrico completo.

Não é difícil verificar que $A \subset X$ tem interior vazio se, e somente se, $A^{c}$ é denso. Assim, para que um espaço topológico $X$ seja um espaço de Baire é necessário e suficiente que toda interseção $S=\cap A_{n}$ de uma família enumerável de abertos $A_{n}$ densos em $X$ seja um subconjunto denso em $X$. Essa propriedade, juntamente com o Teorema de Baire, será fundamental para o desenvolvimento deste trabalho, principalmente no que concerne aos capítulos 2 e 5.

Uma demonstração para o Teorema 2.8 pode ser encontrada em [12, p. 164], a Proposição 2.9 encontra-se demonstrada em [12, p. 163].

\section{PRINCÍPIO DE IDENTIDADE PARA FUNÇÕES ANALÍTICAS}

O próximo teorema é bem interessante e garante que, se duas funções analíticas reais, com mesmo domínio, coincidem em um conjunto com ponto de acumulação no domínio, então elas são iguais.

Teorema 2.10 Sejam $f, g: I \subset \mathbb{R} \longrightarrow \mathbb{R}$ funções analíticas e $X \subset I$ um conjunto com um ponto de acumulação em $I$. Se $f(x)=g(x)$ para todo $x \in X$, então $f=g$.

Uma demonstração para esse teorema pode ser encontrada em [11, p. 403].

\section{$2.2 \quad$ Lemas auxiliares}

O objetivo principal dessa seção é apresentar os lemas que serão utilizados na demonstração do Teorema de Kumar-Thangadurai-Waldschmidt. 
Definição 2.11 Seja $X$ um espaço topológico, dizemos que $G \subset X$ é um subconjunto $G_{\delta}$ de $X$, se $G$ é uma intersecção enumerável de abertos densos em $X$.

Observação 2.12 Alguns livros de Topologia definem conjunto $G_{\delta}$ como uma intersecção enumerável de abertos, não necessariamente densos. Entretanto, tendo em vista os objetivos deste trabalho, vamos considerar a definição como acima.

Exemplo 2.13 O conjunto $\mathbb{R} \backslash \mathbb{Q}$ é um subconjunto $G_{\delta}$ de $\mathbb{R}$, pois,

$$
\mathbb{R} \backslash \mathbb{Q}=\bigcap_{x \in \mathbb{Q}} \mathbb{R} \backslash\{x\}
$$

O próximo lema tem extrema importância para esse capítulo.

Lema 2.14O conjunto dos números de Liouville é um subconjunto $G_{\delta}$ de $\mathbb{R}$.

Demonstração. Mostraremos que $\mathbb{L}=\bigcap_{n \geq 1} U_{n}$, com

$$
U_{n}=\bigcup_{q \geq 2} \bigcup_{p \in \mathbb{Z}}\left(\frac{p}{q}-\frac{1}{q^{n}}, \frac{p}{q}+\frac{1}{q^{n}}\right) \backslash\left\{\frac{p}{q}\right\}
$$

e que cada $U_{n}$ é aberto denso em $\mathbb{R}$. De fato, seja $\xi \in \mathbb{L}$, pelo Lema 1.18, dado $n \geq 1$, existe $\frac{p}{q} \in \mathbb{Q}$, com $q \geq 2$ tal que

$$
\xi \in\left(\frac{p}{q}-\frac{1}{q^{n}}, \frac{p}{q}+\frac{1}{q^{n}}\right) \backslash\left\{\frac{p}{q}\right\}
$$

e, consequentemente,

$$
\xi \in \bigcup_{q \geq 2} \bigcup_{p \in \mathbb{Z}}\left(\frac{p}{q}-\frac{1}{q^{n}}, \frac{p}{q}+\frac{1}{q^{n}}\right) \backslash\left\{\frac{p}{q}\right\} .
$$

Como $n \geq 1$ foi tomado arbitrariamente, temos

$$
\xi \in \bigcap_{n \geq 1}\left(\bigcup_{q \geq 2} \bigcup_{p \in \mathbb{Z}}\left(\frac{p}{q}-\frac{1}{q^{n}}, \frac{p}{q}+\frac{1}{q^{n}}\right) \backslash\left\{\frac{p}{q}\right\}\right)=\bigcap_{n \geq 1} U_{n} .
$$


Reciprocamente, seja $x \in \bigcap_{n \geq 1} U_{n}$. Temos que, para cada $n \geq 1$,

$$
x \in \bigcup_{q \geq 2} \bigcup_{p \in \mathbb{Z}}\left(\frac{p}{q}-\frac{1}{q^{n}}, \frac{p}{q}+\frac{1}{q^{n}}\right) \backslash\left\{\frac{p}{q}\right\} .
$$

Desse modo, para cada $n \geq 1$ existem $q \geq 2$ e $p \in \mathbb{Z}$ tais que

$$
x \in\left(\frac{p}{q}-\frac{1}{q^{n}}, \frac{p}{q}+\frac{1}{q^{n}}\right) \backslash\left\{\frac{p}{q}\right\} .
$$

Isto é, para cada $n \geq 1$ existe $\frac{p}{q} \in \mathbb{Q}$, com $q \geq 2$, tal que

$$
0<\left|x-\frac{p}{q}\right|<\frac{1}{q^{n}}
$$

Portanto, $x \in \mathbb{L}$. Assim, provamos a primeira parte.

Observe que

$$
U_{n}=\bigcup_{q \geq 2} \bigcup_{p \in \mathbb{Z}}\left(\left(\frac{p}{q}-\frac{1}{q^{n}}, \frac{p}{q}+\frac{1}{q^{n}}\right) \bigcap\left(\left\{\frac{p}{q}\right\}^{c}\right)\right),
$$

consequentemente, aberto, para cada $n \geq 1$, já que é uma união enumerável de abertos.

Pela Proposição 2.2,

$$
\overline{U_{n}}=\overline{\bigcup_{q \geq 2} \bigcup_{p \in \mathbb{Z}}\left(\frac{p}{q}-\frac{1}{q^{n}}, \frac{p}{q}+\frac{1}{q^{n}}\right) \backslash\left\{\frac{p}{q}\right\}} \supset \bigcup_{q \geq 2} \bigcup_{p \in \mathbb{Z}} \overline{\left(\frac{p}{q}-\frac{1}{q^{n}}, \frac{p}{q}+\frac{1}{q^{n}}\right) \backslash\left\{\frac{p}{q}\right\}}
$$

para cada $n \geq 1$. E, como

$$
\bigcup_{q \geq 2} \bigcup_{p \in \mathbb{Z}} \overline{\left(\frac{p}{q}-\frac{1}{q^{n}}, \frac{p}{q}+\frac{1}{q^{n}}\right) \backslash\left\{\frac{p}{q}\right\}}=\bigcup_{q \geq 2}\left[\frac{p}{q \in \mathbb{Z}}-\frac{1}{q^{n}}, \frac{p}{q}+\frac{1}{q^{n}}\right] \supset \bigcup_{q \geq 2} \bigcup_{p \in \mathbb{Z}}\left\{\frac{p}{q}\right\},
$$

obtemos, para cada $n \geq 1$,

$$
\overline{U_{n}} \supset \bigcup_{q \geq 2} \bigcup_{p \in \mathbb{Z}}\left\{\frac{p}{q}\right\} \supset \mathbb{Q} .
$$

Como $\mathbb{Q}$ é denso em $\mathbb{R}$, segue que $U_{n}$ é denso, para cada $n \geq 1$. Conclui-se que $\mathbb{L}$ é dado pela intersecção enumerável de abertos densos e, portanto, é um subconjunto $G_{\delta}$ de $\mathbb{R}$. 
Proposição 2.15 Se X é um espaço de Baire, então todo subconjunto $G_{\delta}$ de $X$ é denso.

Demonstração. Seja $G$ um subconjunto $G_{\delta}$ de $X$, assim, $G$ é uma intersecção enumerável de abertos densos em $X$. Se $X$ é um espaço de Baire, então $G$ é denso em $X$.

Corolário 2.16 O conjunto dos números de Liouville é denso em $\mathbb{R}$.

Demonstração. Pelo Lema 2.14, o conjunto dos números de Liouville é $G_{\delta}$ em $\mathbb{R}$, consequentemente, é denso em $\mathbb{R}$, pois $\mathbb{R}$ é um espaço de Baire.

O resultado a seguir é bem intuitivo, entretanto, iremos prová-lo devido a sua utilidade nesse trabalho.

Lema 2.17 Sejam X um espaço topológico e $N$ um conjunto enumerável. Se, para cada $n \in N, G_{n}$ é um subconjunto $G_{\delta}$ de X, então, $\bigcap_{n \in N} G_{n}$ também é subconjunto $G_{\delta}$ de $X$.

Demonstração. Observe que, para cada $n \in N$, existe uma sequência $\left\{A_{n k}\right\}_{k \in \mathbb{N}}$ de abertos densos em $X$ tal que $G_{n}=\bigcap_{k \in \mathbb{N}} A_{n k}$.

Defina a coleção $\beta_{n}=\left\{A_{n k}, k \in \mathbb{N}\right\}$. Assim, $B=\bigcup_{n \in N} \beta_{n}$ é uma união enumerável de coleções enumeráveis, consequentemente, é uma coleção enumerável. Note que $B$ é uma coleção de abertos densos em $X$, sendo assim,

$$
\bigcap_{B_{i} \in B} B_{i}=\bigcap_{n \in N} \bigcap_{k \in \mathbb{N}} A_{n k}
$$

é um subconjunto $G_{\delta}$ de $X$.

Para finalizar a demonstração, é suficiente provar que

$$
\bigcap_{n \in N} G_{n}=\bigcap_{B_{i} \in B} B_{i}
$$


Seja $x \in \bigcap_{n \in N} G_{n}$, temos que $x \in G_{n}$, para todo $n \in N$. Assim, $x \in A_{n k}$, para todo $n \in N$ e para todo $k \in \mathbb{N}$. Portanto,

$$
x \in \bigcap_{n \in N} \bigcap_{k \in \mathbb{N}} A_{n k}=\bigcap_{B_{i} \in B} B_{i}
$$

Reciprocamente, dado $x \in \bigcap_{B_{i} \in B} B_{i}$ temos que $x \in A_{n k}$ para todo $n \in N$ e para todo $k \in \mathbb{N}$. Portanto, $x \in \bigcap_{k \in \mathbb{N}} A_{n k}$, para todo $n \in N$. Consequentemente, $x \in \bigcap_{n \in N} G_{n}$, para todo $n \in N$. O que encerra a demonstração.

Definição 2.18 Sejam $X$ um espaço topológico localmente conexo, $J \subset \mathbb{R}$ um intervalo e $f: X \longrightarrow J$ uma função, diremos que $f$ é nãa-localmente constante $(\boldsymbol{N L C})$ se, para todo aberto conexo não vazio $V \subset X$, a restrição de $f$ a $V$ é não constante.

Exemplo 2.19 Se $J \subset \mathbb{R}$ é um intervalo, então qualquer função $f: I \subset$ $\mathbb{R} \longrightarrow J$ injetiva é $N L C$.

Lema 2.20 Seja X um espaço métrico completo e localmente conexo, J um intervalo em $\mathbb{R}$ e $N$ um conjunto enumerável. Para cada $n \in N$, seja $G_{n}$ um subconjunto $G_{\delta}$ de $J$ e seja $f_{n}: X \longrightarrow J$ uma função contínua que é $N L C$. Então, $\bigcap_{n \in N} f_{n}^{-1}\left(G_{n}\right)$ é um subconjunto $G_{\delta}$ de $X$.

Demonstração. Note que $N$ é enumerável, assim, pelo Lema 2.17 é suficiente provar que para qualquer $n \in N, f_{n}^{-1}\left(G_{n}\right)$ é um subconjunto $G_{\delta}$ de $X$. Observe que, $G_{n}$ é uma interseção enumerável de abertos em $J$, assim, pela continuidade de cada $f_{n}, f_{n}^{-1}\left(G_{n}\right)$ é uma interseção enumerável de abertos em $X$.

Falta mostrar que esses abertos da interseção são densos. Com esse objetivo, mostraremos inicialmente que $f_{n}^{-1}\left(G_{n}\right)$ é denso em $X$, para tanto, utilizaremos a suposição que $f_{n}$ é NLC. 
Seja $V$ um aberto conexo de $X$. Como $f_{n}$ é contínua, $f_{n}(V)$ é conexo em $J$. Além disso, $f_{n}$ é NLC, logo, $f_{n}(V)$ consiste de, no mínimo, dois elementos. Portanto, existe um intervalo $(a, b) \subset J$ com interior não vazio, tal que $(a, b) \subset$ $f_{n}(V)$. Como $J$ é um espaço de Baire, temos que $G_{n}$ é denso em $J$, assim $(a, b) \cap G_{n} \neq \varnothing$. E, daí, $f_{n}(V) \cap G_{n} \neq \varnothing$, o que implica $V \cap f_{n}^{-1}\left(G_{n}\right) \neq \varnothing$. Portanto, $f_{n}^{-1}\left(G_{n}\right)$ é denso em $X$.

Sabemos que $f_{n}^{-1}\left(G_{n}\right)=\bigcap_{k \in \mathbb{N}} A_{k}$, onde cada $A_{k}$ é aberto em $X$. Suponha que existe $i \in \mathbb{N}$ tal que $A_{i}$ não é denso em $X$, isso implica que

$$
f_{n}^{-1}\left(G_{n}\right)=\bigcap_{k \in \mathbb{N}} A_{k} \quad\left(\subset A_{i}\right)
$$

também não é denso em $X$, o que é uma contradição, portanto, $A_{k}$ é denso, para todo $k \in \mathbb{N}$. Isso completa a demonstração.

Proposição 2.21 Seja $X$ um espaço métrico completo (não vazio), sem pontos isolados e seja $E$ um subconjunto $G_{\delta}$ de $X$. Seja $F$ um subconjunto enumerável de E. Então, $E \backslash F$ é um subconjunto $G_{\delta}$ de $X$.

Demonstração. Note que,

$$
E \backslash F=\bigcap_{y \in F}(E \backslash\{y\})
$$

Como $F$ é enumerável, é suficiente mostrar que $E \backslash\{y\}$ é subconjunto $G_{\delta}$ de $X$, para cada $y \in F$.

Sabe-se que,

$$
E=\bigcap_{i \in \mathbb{N}} A_{i}
$$

onde cada $A_{i}$ é aberto denso em $X$. Assim, dado $y \in F$,

$$
E \backslash\{y\}=\bigcap_{i \in \mathbb{N}}\left(A_{i} \backslash\{y\}\right) .
$$


e $A_{i} \backslash\{y\}$ é aberto e denso em $X$, para cada $i \in \mathbb{N}$. Consequentemente, $E \backslash\{y\}$ é subconjunto $G_{\delta}$ de $X$, para cada $y \in F$.

Lema 2.22 Seja $X$ um espaço métrico completo (não vazio), sem pontos isolados. Seja $E$ um subconjunto $G_{\delta}$ de $X$. Então E é não enumerável.

Demonstração. Suponha, por contradição, que $E$ é enumerável, assim, pela Proposição 2.21, $E-E=\varnothing$ é um subconjunto $G_{\delta}$ de $X$. Como $X \neq \varnothing$, temos que $\varnothing$ não é denso em $X$, isso contradiz a Proposição 2.15 .

Corolário 2.23 O conjunto dos números de Liouville é não enumerável.

Demonstração. Pelo Lema 2.14, o conjunto dos números de Liouville é $G_{\delta} \mathrm{em}$ $\mathbb{R}$, consequentemente, é não enumerável, pois $\mathbb{R}$ é um espaço métrico completo (não vazio), sem pontos isolados.

Definição 2.24 As funções $f_{i}: X \subset \mathbb{R} \longrightarrow \mathbb{R}, 0 \leq i \leq n$, são ditas algebricamente dependentes sobre $\mathbb{R}$, se existe um polinômio $P \in \mathbb{R}\left[x_{0}, x_{1}, \ldots, x_{n}\right]$ não constante tal que

$$
P\left(f_{0}(x), f_{1}(x), \ldots, f_{n}(x)\right)=0
$$

para todo $x \in X$. Caso contrário, elas são ditas algebricamente independentes sobre $\mathbb{R}$.

Exemplo 2.25 As funções $f_{0}: \mathbb{R} \longrightarrow \mathbb{R}$ e $f_{1}: \mathbb{R} \longrightarrow \mathbb{R}$, definidas por $f_{0}(x)=x$ e $f_{1}(x)=x^{2}$ são algebricamente dependentes sobre $\mathbb{R}$, basta tomar $P\left(x_{0}, x_{1}\right)=x_{0}^{2}-x_{1}$.

Definição 2.26 As funções $f_{i}: X \subset \mathbb{R} \longrightarrow \mathbb{R}, i \geq 0$, são ditas algebricamente dependentes sobre $\mathbb{R}$ se existe um inteiro $n \geq 0$ tal que as funções $f_{i}: X \subset \mathbb{R} \longrightarrow \mathbb{R}, 1 \leq i \leq n$ são algebricamente dependentes. Caso contrário, elas são ditas algebricamente independentes sobre $\mathbb{R}$. 
Lema 2.27 As funções

$$
x, e^{x}, e^{x^{2}}, \ldots, e^{x^{m}}, \ldots
$$

são algebricamente independentes sobre $\mathbb{R}$.

Demonstração. Sejam $f_{i}: \mathbb{R} \longrightarrow \mathbb{R}, i \geq 0$, definidas por $f_{0}(x)=x$ e $f_{i}(x)=e^{x^{i}}$ para $i \geq 1$. Provaremos que $f_{0}, f_{1}, \ldots, f_{n}$ são algebricamente independentes para todo $n \geq 0$. Usaremos indução.

$\mathrm{O}$ caso $n=0$ é trivial. Seja $n \geq 1$. Suponha que se o resultado é válido para $0,1, \ldots, n-1$. Suponha, por absurdo, que existe $P \in \mathbb{R}\left[x_{0}, x_{1}, \ldots, x_{n}\right]$ não constante de modo que

$$
P\left(x, e^{x}, \ldots, e^{x^{n}}\right) \equiv 0 .
$$

Sem perda de generalidade, podemos supor

$$
P\left(x, e^{x}, \ldots, e^{x^{n}}\right)=a_{0}(x)+a_{1}(x) e^{x^{n}}+\ldots+a_{k}(x) e^{k x^{n}},
$$

para todo $x \in \mathbb{R}$, com $a_{i} \in \mathbb{R}\left[x, e^{x}, \ldots, e^{x^{n-1}}\right]$, para $0 \leq i \leq k$, e $a_{k}(x)$ não identicamente nula. Assim, $a_{0}(x)+a_{1}(x) e^{x^{n}}+\ldots+a_{k}(x) e^{k x^{n}}=0$, para todo $x \in \mathbb{R}$.

Seja $x \in \mathbb{R}_{+}$, como $e^{k x^{n}} \neq 0$ temos

$$
\begin{aligned}
-a_{k}(x) & =\frac{a_{0}(x)}{e^{k x^{n}}}+\frac{a_{1}(x)}{e^{k x^{n}} e^{x^{n}}}+\ldots+\frac{a_{k-1}(x)}{e^{k x^{n}}} e^{(k-1) x^{n}} \\
& =\frac{a_{0}(x)}{e^{k x^{n}}}+\frac{a_{1}(x)}{e^{(k-1) x^{n}}}+\ldots+\frac{a_{k-1}(x)}{e^{x^{n}}} .
\end{aligned}
$$

Utilizando a desigualdade triangular,

$$
\begin{aligned}
0 \leq\left|a_{k}(x)\right| & \leq \frac{\left|a_{0}(x)\right|}{e^{k x^{n}}}+\frac{\left|a_{1}(x)\right|}{e^{(k-1) x^{n}}}+\ldots+\frac{\left|a_{k-1}(x)\right|}{e^{x^{n}}} \\
& \leq \frac{\left|a_{0}(x)\right|}{e^{x^{n}}}+\frac{\left|a_{1}(x)\right|}{e^{x^{n}}}+\ldots+\frac{\left|a_{k-1}(x)\right|}{e^{x^{n}}} .
\end{aligned}
$$

Seja $a \in\left\{a_{0}, a_{1}, \ldots, a_{k-1}\right\}$, assim,

$$
a(x)=\sum_{\left(i_{1}, \ldots, i_{n}\right) \in \mathcal{I}} a_{\left(i_{1}, \ldots, i_{n}\right)} x^{i_{1}}\left(e^{x}\right)^{i_{2}} \ldots\left(e^{x^{n-1}}\right)^{i_{n}}
$$


em que $\mathcal{I}$ é um subconjunto finito de $\{0,1,2, \ldots\}^{n}$ e $a_{\left(i_{1}, \ldots, i_{n}\right)} \in \mathbb{R}$, para cada $\left(i_{1}, \ldots, i_{n}\right) \in \mathcal{I}$, além disso, se $s$ é o grau de $a(x)$, então, $i_{1}+\ldots+i_{n} \leq s$, para cada $\left(i_{1}, \ldots, i_{n}\right) \in \mathcal{I}$, e $i_{j} \leq s$. Em vista disso,

$$
\begin{aligned}
|a(x)| & \leq \sum_{\left(i_{1}, \ldots, i_{n}\right) \in \mathcal{I}}\left|a_{\left(i_{1}, \ldots, i_{n}\right)}\right|\left|x^{i_{1}}\right|\left|\left(e^{x}\right)^{i_{2}}\right| \ldots\left|\left(e^{x^{n-1}}\right)^{i_{n}}\right| \\
& \leq \sum_{\left(i_{1}, \ldots, i_{n}\right) \in \mathcal{I}}\left|a_{\left(i_{1}, \ldots, i_{n}\right)}\right|\left(\left(e^{x^{n-1}}\right)^{s}\right)^{n}=e^{(n s) x^{n-1}} \sum_{\left(i_{1}, \ldots, i_{n}\right) \in \mathcal{I}}\left|a_{\left(i_{1}, \ldots, i_{n}\right)}\right| \\
& =e^{(n s) x^{n-1}} L(a)
\end{aligned}
$$

em que $L(a)=\sum_{\left(i_{1}, \ldots, i_{n}\right) \in \mathcal{I}}\left|a_{\left(i_{1}, \ldots, i_{n}\right)}\right|$ é chamado comprimento de $a$.

Denotaremos por $s_{0}, s_{1}, \ldots, s_{n}$ os graus dos polinômios $a_{0}, a_{1}, \ldots, a_{n}$, respectivamente. Por (2.1) e (2.2),

$$
\begin{aligned}
0 \leq\left|a_{k}(x)\right| & \leq \frac{1}{e^{x^{n}}}\left(\left|a_{0}(x)\right|+\left|a_{1}(x)\right|+\ldots+\left|a_{k-1}(x)\right|\right) \\
& \leq \frac{1}{e^{x^{n}}}\left(L\left(a_{0}\right) e^{\left(n s_{0}\right) x^{n-1}}+L\left(a_{1}\right) e^{\left(n s_{1}\right) x^{n-1}}+\ldots+L\left(a_{k-1}\right) e^{\left(n s_{n}\right) x^{n-1}}\right) .
\end{aligned}
$$

Tome $L=\max \left\{L\left(a_{0}\right), L\left(a_{1}\right), \ldots, L\left(a_{k-1}\right)\right\}$ e $s=\max \left\{s_{0}, s_{1}, \ldots, s_{n}\right\}$. As$\operatorname{sim}$,

$$
0 \leq\left|a_{k}(x)\right| \leq \frac{n \cdot L \cdot e^{(n s) x^{n-1}}}{e^{x^{n}}}=\frac{n \cdot L}{e^{x^{n-1}(-n s+x)}}
$$

e, como

$$
\lim _{x \rightarrow \infty} \frac{n \cdot L}{e^{x^{n-1}(-n s+x)}}=0
$$

temos

$$
\lim _{x \rightarrow \infty}\left|a_{k}(x)\right|=0 .
$$

Portanto, $a_{k}(x)$ é limitada em $\mathbb{R}_{+}$.

Observe que

$$
a_{k}(x)=\sum_{\left(j_{1}, \ldots, j_{n}\right) \in \mathcal{J}} a_{\left(j_{1}, \ldots, j_{n}\right)} x^{j_{1}}\left(e^{x}\right)^{j_{2}} \ldots\left(e^{x^{n-1}}\right)^{j_{n}}
$$


para algum $\mathcal{J}$ subconjunto finito de $\{0,1,2, \ldots\}^{n}$.

Podemos considerar uma ordem total em $\mathcal{J}$ semelhante à ordem lexicográfica com $\left(j_{1}, \ldots, j_{n}\right) \preceq\left(j_{1}^{\prime}, \ldots, j_{n}^{\prime}\right)$ se ocorre uma das seguintes condições:

- $j_{n}<j_{n}^{\prime}$;

- $j_{n}=j_{n}^{\prime}$ e $j_{n-1}<j_{n-1}^{\prime}$;

- $j_{n}=j_{n}^{\prime}, j_{n-1}=j_{n-1}^{\prime}, \ldots, j_{2}=j_{2}^{\prime}, j_{1}<j_{1}^{\prime}$;

- $j_{n}=j_{n}^{\prime}, j_{n-1}=j_{n-1}^{\prime}, \ldots, j_{2}=j_{2}^{\prime}, j_{1}=j_{1}^{\prime}$.

Considerando $\mathcal{J}$ com essa ordem, nós podemos garantir a existência de $\left(i_{1}, \ldots, i_{n}\right) \in \mathcal{J}$ máximo, pois $\mathcal{J}$ é finito. Definimos $\mathcal{K}=\mathcal{J} \backslash\left\{\left(i_{1}, \ldots, i_{n}\right)\right\}$.

É importante observar que, dado $\left(j_{1}, \ldots, j_{n}\right) \in \mathcal{K}$, temos $\left(j_{1}, \ldots, j_{n}\right) \preceq$ $\left(i_{1}, \ldots, i_{n}\right)$, com $\left(j_{1}, \ldots, j_{n}\right)$ e $\left(i_{1}, \ldots, i_{n}\right) n$-uplas distintas. Assim, garantimos a existência de $l \in\{1, \ldots, n\}$ tal que $j_{l}<i_{l}$ e $j_{m}=i_{m}$, para $m \in\{l+1, \ldots, n\}$. Note que,

$a_{k}(x)=a_{\left(i_{1}, \ldots, i_{n}\right)} x^{i_{1}}\left(e^{x}\right)^{i_{2}} \ldots\left(e^{x^{n-1}}\right)^{i_{n}}+\sum_{\left(j_{1}, \ldots, j_{n}\right) \in \mathcal{K}} a_{\left(j_{1}, \ldots, j_{n}\right)} x^{j_{1}}\left(e^{x}\right)^{j_{2}} \ldots\left(e^{x^{n-1}}\right)^{j_{n}}$.

Tome

$$
A(x)=\left|a_{\left(i_{1}, \ldots, i_{n}\right)} x^{i_{1}}\left(e^{x}\right)^{i_{2}} \ldots\left(e^{x^{n-1}}\right)^{i_{n}}\right|
$$

e

$$
B(x)=\left|\sum_{\left(j_{1}, \ldots, j_{n}\right) \in \mathcal{K}} a_{\left(j_{1}, \ldots, j_{n}\right)} x^{j_{1}}\left(e^{x}\right)^{j_{2}} \ldots\left(e^{x^{n-1}}\right)^{j_{n}}\right| .
$$

Observe que $\lim _{x \longrightarrow+\infty} A(x)=+\infty$, já que $a_{k}(x)$ é não identicamente nula. Mostraremos que $\lim _{x \longrightarrow+\infty} \frac{B(x)}{A(x)}=0$.

De fato, se $B(x)$ é identicamente nula, temos $\lim _{x \longrightarrow+\infty} \frac{B(x)}{A(x)}=0$. Vamos supor $B(x)$ não identicamente nula. Note que,

$$
\frac{B(x)}{A(x)}=\left|\sum_{\left(j_{1}, \ldots, j_{n}\right) \in \mathcal{K}} \frac{a_{\left(j_{1}, \ldots, j_{n}\right)} x^{j_{1}}\left(e^{x}\right)^{j_{2}} \ldots\left(e^{x^{n-1}}\right)^{j_{n}}}{a_{\left(i_{1}, \ldots, i_{n}\right)} x^{i_{1}}\left(e^{x}\right)^{i_{2}} \ldots\left(e^{x^{n-1}}\right)^{i_{n}}}\right| .
$$


Seja $\left(j_{1}, \ldots, j_{n}\right) \in \mathcal{K}$ e $m \in\{1, \ldots, n\}$ tal que $j_{n}=i_{n}, \ldots, j_{m+1}=i_{m+1}$ e $j_{m}<i_{m}$. Assim,

$$
\begin{aligned}
& \lim _{x \longrightarrow+\infty} \frac{a_{\left(j_{1}, \ldots, j_{n}\right)} x^{j_{1}}\left(e^{x}\right)^{j_{2}} \ldots\left(e^{x^{n-1}}\right)^{j_{n}}}{a_{\left(i_{1}, \ldots, i_{n}\right)} x^{i_{1}}\left(e^{x}\right)^{i_{2}} \ldots\left(e^{x^{n}-1}\right)^{i_{n}}}= \\
& \frac{a_{\left(j_{1}, \ldots, j_{n}\right)}}{a_{\left(i_{1}, \ldots, i_{n}\right)}} \lim _{x \longrightarrow+\infty} x^{j_{1}-i_{1}}\left(e^{x}\right)^{j_{2}-i_{2}} \ldots\left(e^{x^{m-2}}\right)^{j_{m-1}-i_{m-1}}\left(e^{x^{m-1}}\right)^{j_{m}-i_{m}}= \\
& \frac{a_{\left(j_{1}, \ldots, j_{n}\right)}}{a_{\left(i_{1}, \ldots, i_{n}\right)}} \lim _{x \longrightarrow+\infty} e^{\left(j_{1}-i_{1}\right) \log x+\left(j_{2}-i_{2}\right) x+\ldots+\left(j_{m-1}-i_{m-1}\right) x^{m-2}+\left(j_{m}-i_{m}\right) x^{m-1}}=0 .
\end{aligned}
$$

Portanto,

$$
0 \leq \lim _{x \longrightarrow+\infty} \frac{B(x)}{A(x)} \leq \sum_{\left(j_{1}, \ldots, j_{n}\right) \in \mathcal{K}} \lim _{x \longrightarrow+\infty}\left|\frac{a_{\left(j_{1}, \ldots, j_{n}\right)} x^{j_{1}}\left(e^{x}\right)^{j_{2}} \ldots\left(e^{x^{n-1}}\right)^{j_{n}}}{a_{\left(i_{1}, \ldots, i_{n}\right)} x^{i_{1}}\left(e^{x}\right)^{i_{2}} \ldots\left(e^{x^{n-1}}\right)^{i_{n}}}\right|=0 .
$$

Como $B(x) / A(x)$ é contínua para $x$ suficientemente grande, segue, pelo Teorema do confronto, que

$$
\lim _{x \rightarrow+\infty} \frac{B(x)}{A(x)}=0
$$

E, pela Proposição 2.4,

$$
\lim _{x \longrightarrow+\infty} A(x)-B(x)=+\infty .
$$

Entretanto,

$$
A(x)-B(x) \leq\left|a_{k}(x)\right|
$$

e, assim, concluímos que $a_{k}$ não pode ser limitada em $\mathbb{R}_{+}$e isso contradiz sua limitação.

Lema 2.28 Seja $\psi: \mathbb{R} \longrightarrow \mathbb{R}$ uma função analítica e não identicamente nula, então, $Z(\psi)=\{x \in \mathbb{R} \mid \psi(x)=0\}$ é enumerável. 
Demonstração. Suponha que $Z(\psi)=\{x \in \mathbb{R} \mid \psi(x)=0\}$ é não enumerável, assim, pela Proposição 2.3, $Z(\psi)$ tem ponto de acumulação. Observe que $\psi(x)=0$ para todo $x \in Z(\psi)$, logo, pelo Princípio de Identidade para Funções Analíticas (Teorema 2.10), $\psi(x)=0$ para todo $x \in \mathbb{R}$, o que contradiz o fato de que $\psi$ é uma função não identicamente nula. Segue que $Z(\psi)=\{x \in \mathbb{R} \mid$ $\psi(x)=0\}$ é enumerável.

O lema a seguir segue diretamente da Proposição 1.13.

Lema 2.29 Se $\xi$ é um número de Liouville, então, $\xi, \xi^{2}, \ldots, \xi^{n}$ são números de Liouville linearmente independentes sobre $\mathbb{Q}$, para todo $n \in \mathbb{N}$.

\subsection{Prova do teorema}

Teorema 2.30 (Kumar-Thangadurai-Waldschmidt) Sejam $m$ e $n$ inteiros tais que $1 \leq m \leq n$. Então existe uma quantidade não enumerável de $n$-uplas $\left(\alpha_{1}, \ldots, \alpha_{n}\right) \in \mathbb{L}^{n}$ tais que $\alpha_{1}, \ldots, \alpha_{n}$ são linearmente independentes sobre $\mathbb{Q}, e^{\alpha_{i}} \in \mathbb{L}$ para todo $i=1,2 \ldots, n$ e

$$
\operatorname{grtr}\left(\mathbb{Q}\left(\alpha_{1}, \ldots, \alpha_{n}, e^{\alpha_{1}}, \ldots, e^{\alpha_{n}}\right) \mid \mathbb{Q}\right)=n+m
$$

Demonstração. Sejam $n$ e $m$ inteiros tais que $1 \leq m \leq n$. Provaremos a afirmação por indução sobre $m$.

Assuma $m=1$. Provaremos o resultado para todo $n \geq 1$.

Para cada polinômio não nulo

$$
P\left(x_{0}, x_{1}, \ldots, x_{n}\right) \in \mathbb{Q}\left[x_{0}, x_{1}, \ldots, x_{n}\right],
$$

defina a função analítica

$$
f_{P}: \mathbb{R} \longrightarrow \mathbb{R} \text { por } f_{P}(x)=P\left(x, e^{x}, \ldots, e^{x^{n}}\right) .
$$

Além disso, defina 


$$
Z\left(f_{P}\right)=\left\{x \in \mathbb{R} \mid f_{P}(x)=0\right\}
$$

Observe que, pelo Lema 2.27, $f_{P}$ não é identicamente nula, assim, pelo Lema 2.28, $Z\left(f_{p}\right)$ é enumerável. Em vista disso,

$$
\mathbb{R} \backslash Z\left(f_{P}\right)=\bigcap_{x \in Z\left(f_{P}\right)} \mathbb{R} \backslash\{x\}
$$

é um subconjunto $G_{\delta}$ de $\mathbb{R}$. Segue, pelo Lema 2.17, que,

$$
F=\bigcap_{P \in \mathbb{Q}\left[X_{0}, \ldots, X_{n}\right] \backslash\{0\}}\left(\mathbb{R} \backslash Z\left(f_{P}\right)\right)
$$

é subconjunto $G_{\delta}$ de $\mathbb{R}$. Agora, defina

$$
G=\left\{\alpha \in \mathbb{L} \mid e^{\alpha^{j}} \in \mathbb{L} \text { para } j=1, \ldots, n\right\} .
$$

Mostraremos que $G$ é $G_{\delta}$ em $\mathbb{R}$. De fato, defina $h_{k}: \mathbb{R} \longrightarrow \mathbb{R}, k \in \mathbb{N}$, como segue:

$$
h_{1}(x)=e^{x}, h_{2}(x)=e^{x^{2}}, \ldots, h_{n}(x)=e^{x^{n}}, \quad h_{k}(x)=x, \text { se } k>n .
$$

Pelo Lema 2.20,

$$
\bigcap_{k \geq 1} h_{k}^{-1}(\mathbb{L})
$$

é $G_{\delta}$ em $\mathbb{R}$.

AFIRMAÇÃO 1: $G=\bigcap_{k \geq 1} h_{k}^{-1}(\mathbb{L})$

De fato, se $\alpha \in G$, então $e^{\alpha^{j}} \in \mathbb{L}$ para $j=1, \ldots, n$. Assim, $\alpha=h_{k}^{-1}\left(e^{\alpha^{k}}\right) \in$ $h_{k}^{-1}(\mathbb{L})$, para cada $k=1, \ldots, n$. Além disso, $\alpha=h_{k}^{-1}(\alpha) \in h_{k}^{-1}(\mathbb{L})$, para $k>n$. Logo, $\alpha \in \bigcap_{k \geq 1} h_{k}^{-1}(\mathbb{L})$.

Por outro lado, se $\alpha \in \bigcap_{k \geq 1} h_{k}^{-1}(\mathbb{L})$. Temos $h_{k}(\alpha) \in \mathbb{L}$, para cada $k \geq 1$, assim,

$$
\alpha, e^{\alpha}, e^{\alpha^{2}}, \ldots, e^{\alpha^{n}} \in \mathbb{L}
$$


Logo, $\alpha \in G$. Portanto, a Afirmação 1 está provada.

Segue que $G$ é $G_{\delta}$ em $\mathbb{R}$, consequentemente, pelo Lema 2.17, $E=F \cap G$ é subconjunto $G_{\delta}$ de $\mathbb{R}$, e assim, pelo Lema $2.22, E$ é não enumerável.

Além disso, por construção, dado $\alpha \in E$, os números $\alpha, e^{\alpha}, e^{\alpha^{2}}, \ldots, e^{\alpha^{n}}$ estão em $\mathbb{L}$ e são algebricamente independentes sobre $\mathbb{Q}$ e, pelo Lema 2.29, $\alpha, \alpha^{2}, \ldots, \alpha^{n}$ são todos números de Liouville linearmente independentes sobre Q. Assim,

$$
\operatorname{grtr}\left(\mathbb{Q}\left(\alpha, \alpha^{2}, \ldots, \alpha^{n}, e^{\alpha}, e^{\alpha^{2}}, \ldots, e^{\alpha^{n}}\right) \mid \mathbb{Q}\right)=n+1
$$

Com isso, concluímos que a afirmação vale para $m=1$ e para todo $n \geq 1$. Essa é nossa base de indução.

Como hipótese de indução, vamos supor que a afirmação é válida para $m-1$ e para todo $n \geq m-1$. Desse modo, dado $n \geq m-1$, existe uma quantidade não enumerável de $n$-uplas $\left(\gamma_{1}, \ldots, \gamma_{n}\right) \in \mathbb{L}^{n}$, tais que $\gamma_{1}, \ldots, \gamma_{n}$ são linearmente independentes sobre $\mathbb{Q}, e^{\gamma_{i}} \in \mathbb{L}$, para todo $i \in\{1, \ldots, n\}$ e

$$
\operatorname{grtr}\left(\mathbb{Q}\left(\gamma_{1}, \ldots, \gamma_{n}, e_{1}^{\gamma}, \ldots, e^{\gamma_{n}}\right) \mid \mathbb{Q}\right)=n+m-1
$$

Queremos mostrar que a afirmação é válida para $m$, isto é, queremos mostrar que para todo $n \geq m$, existe uma quantidade não enumerável de $n$-uplas $\left(\alpha_{1}, \ldots, \alpha_{n}\right) \in \mathbb{L}^{n}$ tais que $\alpha_{1}, \ldots, \alpha_{n}$ são linearmente independentes sobre $\mathbb{Q}$, $e^{\alpha_{i}} \in \mathbb{L}$ para todo $i \in\{1, \ldots, n\}$ e

$$
\operatorname{grtr}\left(\mathbb{Q}\left(\alpha_{1}, \ldots, \alpha_{n}, e^{\alpha_{1}}, \ldots, e^{\alpha_{n}}\right) \mid \mathbb{Q}\right)=n+m
$$

Note que, $n \geq m$ implica $n-1 \geq m-1$, assim, pela hipótese de indução, há uma quantidade não enumerável de $(n-1)$-uplas $\left(\alpha_{1}, \ldots, \alpha_{n-1}\right) \in \mathbb{L}^{n-1}$ tais que $\alpha_{1}, \ldots, \alpha_{n-1}$ são linearmente independentes sobre $\mathbb{Q}, e^{\alpha_{1}}, \ldots, e^{\alpha_{n-1}}$ são números de Liouville e

$$
\operatorname{grtr}\left(\mathbb{Q}\left(\alpha_{1}, \ldots, \alpha_{n-1}, e^{\alpha_{1}}, \ldots, e^{\alpha_{n-1}}\right) \mid \mathbb{Q}\right)=(n-1)+(m-1)=n+m-2
$$


Escolha uma $(n-1)$-upla $\left(\alpha_{1}, \ldots, \alpha_{n-1}\right)$.

Considere o subconjunto $E$ de $\mathbb{R}$ que consiste de todos $\alpha \in \mathbb{R}$ tais que o conjunto $\left\{\alpha, e^{\alpha}\right\}$ é algebricamente independente sobre

$$
\mathbb{Q}\left(\alpha_{1}, \ldots, \alpha_{n-1}, e^{\alpha_{1}}, \ldots, e^{\alpha_{n-1}}\right) .
$$

Se

$$
P(x, y) \in \mathbb{Q}\left(\alpha_{1}, \ldots, \alpha_{n-1}, e^{\alpha_{1}}, \ldots, e^{\alpha_{n-1}}\right)[x, y]
$$

é um polinômio não constante, defina a função analítica $f_{P}(x)=P\left(x, e^{x}\right)$ em $\mathbb{R}$. Sabemos, pelo Lema 2.27, que $x, e^{x}$ são funções algebricamente independentes sobre $\mathbb{R}$, assim, se $P$ é um polinômio não nulo, temos $f_{P}$ uma função não identicamente nula. Portanto, pelo Lema 2.28, o conjunto de zeros de $f_{P}$ em $\mathbb{R}$ é enumerável. Além disso, há somente uma quantidade enumerável de polinômios $P(x, y)$ com coeficientes no corpo

$$
\mathbb{Q}\left(\alpha_{1}, \ldots, \alpha_{n-1}, e^{\alpha_{1}}, \ldots, e^{\alpha_{n-1}}\right),
$$

sendo assim, podemos garantir que é enumerável o conjunto

$$
\mathbb{R} \backslash E=\underset{P(x, y) \in \mathbb{Q}\left(\alpha_{1}, \ldots, \alpha_{n-1}, e^{\left.\alpha_{1}, \ldots, e^{\alpha_{n-1}}\right)[x, y] \backslash\{0\}}\right.}{\bigcup_{P}} Z\left(f_{P}\right) .
$$

Consequentemente,

$$
E=\bigcap_{x \in \mathbb{R} \backslash E} \mathbb{R} \backslash\{x\}
$$

é um subconjunto $G_{\delta}$ de $\mathbb{R}$.

Como $\mathbb{L}$ é um subconjunto $G_{\delta}$ de $\mathbb{R}$, então $E \cap \mathbb{L}$ é um subconjunto $G_{\delta}$ de $\mathbb{R}$ e, portanto, é não enumerável.

Note que, para cada $\alpha \in E \cap \mathbb{L}$, o conjunto $\left\{\alpha, e^{\alpha}\right\}$ é algebricamente independente sobre

$$
\mathbb{Q}\left(\alpha_{1}, \ldots, \alpha_{n-1}, e^{\alpha_{1}}, \ldots, e^{\alpha_{n-1}}\right) .
$$


Concluímos que

$$
\operatorname{grtr}\left(\mathbb{Q}\left(\alpha_{1}, \ldots, \alpha_{n-1}, \alpha, e^{\alpha_{1}}, \ldots, e^{\alpha_{n-1}}, e^{\alpha}\right) \mid \mathbb{Q}\right)=n+m
$$

O que completa a prova do teorema.

Como vimos, a propriedade $G_{\delta}$ do conjunto dos números de Liouville foi de extrema importância para a verificação do Teorema de Kumar-ThangaduraiWaldschmidt. No Capítulo 6, provaremos outros resultados interessantes que decorrem dessa propriedade. 


\section{Capítulo 3}

\section{Sobre Decomposições de}

\section{Liouville}

Um dos principais resultados em Teoria Elementar dos Números é o Teorema Fundamental da Aritmética. Esse teorema garante que qualquer número natural $n$, maior que 1 , pode ser escrito, de maneira única, como produto de potências de primos. Existem outros resultados importantes sobre decomposição em Matemática como, por exemplo, o Teorema Fundamental dos Grupos Abelianos Finitamente Gerados, que garante a decomposição de um grupo abeliano finitamente gerado como uma soma direta de grupos cíclicos. Neste capítulo, falaremos sobre decomposições de números reais em números de Liouville.

Em 1962, Erdös provou que todo número real pode ser escrito como soma de dois números de Liouville, ou seja, dada $f: \mathbb{R}^{2} \longrightarrow \mathbb{R}$, definida por $f(x, y)=$ $x+y$, e dado $\alpha \in \mathbb{R}$, existem números de Liouville $\sigma, \tau$, tais que $f(\sigma, \tau)=\alpha$. Esse é um resultado bastante interessante e falaremos um pouco mais sobre ele na Seção 3.1.

O principal objetivo deste capítulo é generalizar esse Teorema de Erdös, apresentando uma classe mais geral de funções $f: \mathbb{R}^{2} \longrightarrow \mathbb{R}$, de modo que, dado $\alpha \in \mathbb{R}$ (satisfazendo determinadas condições), existam números de Liou- 
ville $\sigma, \tau$, tais que $f(\sigma, \tau)=\alpha$. Faremos isso na Seção 3.2, com base no Artigo On Liouville decompositions in Local Fields de Edward B. Burger.

\subsection{Um teorema de Erdös}

Não é difícil provar que qualquer número real pode ser decomposto como soma de dois números transcendentes, contudo, esse não é um resultado tão interessante, visto que quase todo número real é transcendente.

Nesta seção, veremos um resultado a priori surpreendente: qualquer número real pode ser escrito como soma de dois números de Liouville. Esse resultado é bem interessante, uma vez que o conjunto dos números de Liouville tem medida nula em $\mathbb{R}$, ou seja, quase nenhum número real é de Liouville. Segundo Marques (Ver [18, p. 86]), podemos pensar então que, mesmo sendo um conjunto "invisível", os números de Liouville estão estrategicamente posicionados na reta real.

Erdös provou esse resultado em 1962, no artigo Representations of real numbers as sums and products of Liouville numbers. Nesse artigo, ele deu uma prova construtiva, onde os números de Liouville são explicitados, e uma prova não construtiva, em que ele utiliza as propriedades de conjunto $G_{\delta}$. Tais demonstrações serão apresentadas aqui, com algumas adaptações.

Primeiramente, mostraremos que qualquer número real pode ser escrito como soma de dois elementos de um subconjunto $G_{\delta}$ de $\mathbb{R}$.

Lema 3.1 Se $G \subset \mathbb{R}$ é um subconjunto $G_{\delta}$, então dado $\alpha \in \mathbb{R}$, existem $x, y \in$ $G$ tais que $x+y=\alpha$.

Demonstração. Sabemos que

$$
G=\bigcap_{n \in \mathbb{N}} A_{n}
$$

onde $A_{n}$ é aberto denso em $\mathbb{R}$, para cada $n$. 
AFIRMAÇ $\tilde{A} O$ 1: $\alpha-G=\{\alpha-s \mid s \in G\}$ é um subconjunto $G_{\delta}$ de $\mathbb{R}$.

Inicialmente, mostraremos que

$$
\alpha-G=\bigcap_{n \in \mathbb{N}}\left(\alpha-A_{n}\right)
$$

em que $\alpha-A_{n}=\left\{\alpha-s \mid s \in A_{n}\right\}$. Note que, dado $t \in \alpha-G$, existe $s \in G$, tal que $t=a-s$. Como $s \in G$, tem-se $s \in A_{n}$, para todo $n \in \mathbb{N}$, e assim, $t=\alpha-s \in \alpha-A_{n}$, para todo $n \in \mathbb{N}$. Logo,

$$
t \in \bigcap_{n \in \mathbb{N}}\left(\alpha-A_{n}\right)
$$

Reciprocamente, dado

$$
t \in \bigcap_{n \in \mathbb{N}}\left(\alpha-A_{n}\right)
$$

tem-se $t \in \alpha-A_{n}$, para cada $n \in \mathbb{N}$. Assim, para cada $n \in \mathbb{N}$, existe $s_{n} \in A_{n}$ tal que $t=\alpha-s_{n}$. Pela unicidade do inverso aditivo, $s_{i}=s_{j}$ mesmo que $i \neq j$, defina $s=s_{1}$. Desse modo,

$$
t=\alpha-s \in \alpha-\bigcap_{n \in \mathbb{N}} A_{n}=\alpha-G
$$

Agora, observe que $A_{n}$ é aberto e denso para cada $n \in \mathbb{N}$, desse modo, $\alpha-A_{n}$ é aberto e denso, consequentemente $\alpha-G$ é $G_{\delta}$. E a Afirmação 1 está provada.

Como $G$ e $\alpha-G$ são conjuntos $G_{\delta}$, então, $G \cap(\alpha-G)$ é $G_{\delta}$ e, consequentemente, não vazio. Conclui-se que, dado $\alpha \in \mathbb{R}$ existe $y \in G \cap(\alpha-G)$. Desse modo, $y \in G$ e $y \in \alpha-G$. Assim, existe $x \in G$ tal que $y=\alpha-x$.

Portanto, existem $x, y \in G$ tais que $x+y=\alpha$, como queríamos demonstrar.

Teorema 3.2 (Erdös) Seja $f: \mathbb{R}^{2} \longrightarrow \mathbb{R}$ definida por $f(x, y)=x+y$. Dado $\alpha \in \mathbb{R}$, existem números de Liouville $\sigma$ e $\tau$ tais que $f(\sigma, \tau)=\alpha$. 
$1^{\text {a }}$ Demonstração. Sabemos que $\mathbb{L}$ é um subconjunto $G_{\delta}$ de $\mathbb{R}$. Segue, do Lema 3.1, que, dado $\alpha \in \mathbb{R}$, existem $\sigma, \tau \in \mathbb{L}$ tais que $\sigma+\tau=\alpha$.

$2^{\text {a }}$ Demonstração. Se $\alpha \in \mathbb{Q}$, escolhemos $\sigma$ um número de Liouville qualquer, fixado. Pela Proposição 1.13, $\tau=\alpha-\sigma$ também é número de Liouville e

$$
f(\sigma, \tau)=\sigma+\tau=\sigma+(\alpha-\sigma)=\alpha .
$$

Se $\alpha \notin \mathbb{Q}$, temos $\alpha=\lfloor\alpha\rfloor+\{\alpha\}$, onde $\lfloor\alpha\rfloor \in \mathbb{Z}$ corresponde à parte inteira de $\alpha$ e $\{\alpha\} \in(0,1)$ corresponde à parte fracionária. É suficiente provar que existem números de Liouville $\tau_{1}$ e $\tau_{2}$ tais que $\tau_{1}+\tau_{2}=\{\alpha\}$. Pois, tomando $\sigma=\lfloor\alpha\rfloor+\tau_{1}$ e $\tau=\tau_{2}$, obtemos $f(\sigma, \tau)=\sigma+\tau=\lfloor\alpha\rfloor+\tau_{1}+\tau_{2}=\lfloor\alpha\rfloor+\{\alpha\}=\alpha$. Como $\{\alpha\} \in(0,1)$, podemos escrever sua expansão 2-ádica como

$$
\{\alpha\}=\sum_{k=1}^{\infty} \frac{\varepsilon_{k}}{2^{k}}
$$

$\operatorname{com} \varepsilon_{k} \in\{0,1\}$. Em seguida, definimos

$$
\tau_{1}=\sum_{k=1}^{\infty} \frac{\alpha_{k}}{2^{k}} \text { e } \tau_{2}=\sum_{k=1}^{\infty} \frac{\beta_{k}}{2^{k}}
$$

onde para $n ! \leq k<(n+1)$ ! temos

$$
\begin{aligned}
& \alpha_{k}=\varepsilon_{k} \text { e } \beta_{k}=0 \text { se } n \notin 2 \mathbb{Z}, \\
& \alpha_{k}=0 \text { e } \beta_{k}=\varepsilon_{k} \text { se } n \in 2 \mathbb{Z} .
\end{aligned}
$$

Observe que $\tau_{1}+\tau_{2}=\{\alpha\}$. Verificaremos apenas que $\tau_{2}$ é número de Liouville, pois, para $\tau_{1}$ o argumento é análogo.

Dado $n \geq 1$, sejam

$$
q_{n}=2^{(2 n+1) !-1} \text { e } p_{n}=q_{n}\left(\sum_{k=1}^{(2 n+1) !-1} \frac{\beta_{k}}{2^{k}}\right) .
$$

Assim,

$$
\left|\tau_{2}-\frac{p_{n}}{q_{n}}\right|=\sum_{k=(2 n+1) !}^{\infty} \frac{\beta_{k}}{2^{k}}
$$


Note que, $\beta_{k}=0$ para $(2 n+1) ! \leq k<(2 n+2)$ !. Desse modo,

$$
\left|\tau_{2}-\frac{p_{n}}{q_{n}}\right|=\sum_{k=(2 n+2) !}^{\infty} \frac{\beta_{k}}{2^{k}} \leq \sum_{k=(2 n+2) !}^{\infty} \frac{1}{2^{k}}=\frac{1}{2^{(2 n+2) !-1}}<\frac{1}{2^{n(2 n+1) !-n}}=\frac{1}{q_{n}^{n}} .
$$

Conclui-se que $\tau_{2}$ é um número de Liouville.

\subsection{Teorema de decomposição}

Nesta seção apresentaremos o Teorema de decomposição, que é o foco principal deste capítulo.

Esse teorema garante que alguns números reais podem ser decompostos como $f(\sigma, \tau)$, onde $\sigma$ e $\tau$ são números de Liouville, para uma classe muito grande de funções $f(x, y)$.

Definição 3.3 Se $Z \subset \mathbb{R}^{2}$ é um subconjunto aberto, $f: Z \longrightarrow \mathbb{R}$ uma função contínua e $\alpha \in \mathbb{R}$, então dizemos que $f$ é localmente injetiva em $\alpha$ se existem conjuntos abertos $U$ e $V$ em $\mathbb{R}, U \times V \subset Z$, de modo que:

(i) Para todo $x \in U$, existe um único $y \in V$ de modo que $f(x, y)=\alpha$;

(ii) Para todo $y \in V$, existe um único $x \in U$ de modo que $f(x, y)=\alpha$.

Mais precisamente, dizemos que $f$ é localmente injetiva em $\alpha$ sobre $U \times V$.

Teorema 3.4 (Teorema de decomposição) Sejam $X \subset \mathbb{R}^{2}$ um conjunto aberto, $f: X \longrightarrow \mathbb{R}$ uma função contínua e $\alpha \in \mathbb{R}$. Suponha que $f$ é localmente injetiva em a sobre $U_{1} \times V_{1}$ e que $g_{1}: U_{1} \longrightarrow V_{1}$ e $g_{2}: V_{1} \longrightarrow U_{1}$, definidas implicitamente por $f\left(x, g_{1}(x)\right)=\alpha$ e $f\left(g_{2}(y), y\right)=\alpha$, são aplicações abertas. Então, existem números de Liouville $\sigma$ e $\tau$ tais que

$$
f(\sigma, \tau)=\alpha
$$

Lema 3.5 Nas hipóteses do Teorema de decomposição, g $g_{1}$ é um homeomorfismo e $g_{2}=g_{1}^{-1}$. 
Demonstração. Observe que $g_{1}$ é sobrejetiva, pois dado $y \in V_{1}$, existe $x \in$ $U_{1}$ tal que $f(x, y)=\alpha$, $\log 0 g_{1}(x)=y$. Para a injetividade, suponha, por absurdo, que existem $x_{1}, x_{2} \in U_{1}$ distintos tais que $g_{1}\left(x_{1}\right)=g_{1}\left(x_{2}\right)=y \in V_{1}$, assim $f\left(x_{1}, y\right)=f\left(x_{2}, y\right)=\alpha$, o que contradiz a injetividade local de $f$ em $\alpha$ sobre $U_{1} \times V_{1}$. Portanto, $g_{1}: U_{1} \longrightarrow V_{1}$ é bijetiva, logo, existe $g^{-1}: V_{1} \longrightarrow$ $U_{1}$. Suponha, por absurdo, que existe $y \in V_{1}$ tal que $g_{2}(y) \neq g_{1}^{-1}(y)$. Por construção, $f\left(g_{2}(y), y\right)=f\left(g_{1}^{-1}(y), y\right)=\alpha$, entretanto, $g_{2}(y), g_{1}^{-1}(y) \in U_{1}$ são distintos, o que contradiz a injetividade local de $f$ em $\alpha$ sobre $U_{1} \times V_{1}$, logo, $g_{2}=g_{1}^{-1}$. Além disso, $g_{1}$ e $g_{1}^{-1}$ são contínuas, uma vez que, por hipótese, $g_{1}$ e $g_{2}$ são aplicações abertas.

Demonstração do Teorema de decomposição. Podemos supor, sem perda de generalidade, que $U_{1}$ e $V_{1}$ são conexos, de modo que $\overline{U_{1}} \times \overline{V_{1}} \subset X$. Como $\mathbb{Q}$ é denso em $\mathbb{R}$, podemos escolher $\frac{a_{1}}{b_{1}} \in U_{1} \cap \mathbb{Q}$. Observe que,

$$
U_{1} \cap\left(\frac{a_{1}}{b_{1}}-\frac{1}{b_{1}}, \frac{a_{1}}{b_{1}}+\frac{1}{b_{1}}\right)
$$

é aberto em $\mathbb{R}$, contendo $\frac{a_{1}}{b_{1}}$. Logo, existe $\varepsilon_{1}>0$ tal que

$$
W_{1}=\left(\frac{a_{1}}{b_{1}}-\varepsilon_{1}, \frac{a_{1}}{b_{1}}+\varepsilon_{1}\right) \subset U_{1} \cap\left(\frac{a_{1}}{b_{1}}-\frac{1}{b_{1}}, \frac{a_{1}}{b_{1}}+\frac{1}{b_{1}}\right) .
$$

Por hipótese, $g_{1}$ é uma aplicação aberta, logo, $Z_{1}=g_{1}\left(W_{1}\right) \subseteq V_{1}$ é aberto e $f$ é localmente injetiva em $\alpha$ sobre $W_{1} \times Z_{1}$. Selecionamos $\frac{c_{1}}{d_{1}} \in \mathbb{Q} \cap Z_{1}$, em seguida, selecionamos $u_{1} \in W_{1}$ tal que $f\left(u_{1}, \frac{c_{1}}{d_{1}}\right)=\alpha$. Observe que

$$
Z_{1} \cap\left(\frac{c_{1}}{d_{1}}-\frac{1}{d_{1}}, \frac{c_{1}}{d_{1}}+\frac{1}{d_{1}}\right)
$$

é aberto, contendo $\frac{c_{1}}{d_{1}}$. Logo, existe $\delta_{1}>0$ tal que

$$
V_{2}=\left(\frac{c_{1}}{d_{1}}-\delta_{1}, \frac{c_{1}}{d_{1}}+\delta_{1}\right) \subset Z_{1} \cap\left(\frac{c_{1}}{d_{1}}-\frac{1}{d_{1}}, \frac{c_{1}}{d_{1}}+\frac{1}{d_{1}}\right) .
$$


Segue do lema anterior que $U_{2}=g_{1}^{-1}\left(V_{2}\right)$ é aberto, contido em $W_{1}$, e $f$ é localmente injetiva em $\alpha$ sobre $U_{2} \times V_{2}$.

Seja $N \geq 2$. Assumindo que

$$
\left\{\frac{a_{1}}{b_{1}}, \frac{a_{2}}{b_{2}}, \ldots, \frac{a_{N-1}}{b_{N-1}}\right\} \subset \mathbb{Q},\left\{\frac{c_{1}}{d_{1}}, \frac{c_{2}}{d_{2}}, \ldots, \frac{c_{N-1}}{d_{N-1}}\right\} \subset \mathbb{Q}
$$

e abertos $U_{N}, V_{N}$ em $\mathbb{R}$, com $f$ localmente injetiva em $\alpha$ sobre $U_{N} \times V_{N}$, agora construiremos $\frac{a_{N}}{b_{N}}$ e $\frac{c_{N}}{d_{N}}$.

Seja $\frac{a_{N}}{b_{N}}$ um ponto de $\mathbb{Q}$ tal que

$$
\frac{a_{N}}{b_{N}} \in U_{N} \backslash\left\{\frac{a_{1}}{b_{1}}, \frac{a_{2}}{b_{2}}, \ldots, \frac{a_{N-1}}{b_{N-1}}\right\}
$$

Observe que

$$
U_{N} \cap\left(\frac{a_{N}}{b_{N}}-\frac{1}{b_{N}^{N}}, \frac{a_{N}}{b_{N}}+\frac{1}{b_{N}^{N}}\right)
$$

é aberto, contendo $\frac{a_{N}}{b_{N}}$. Logo, existe $\varepsilon_{N}>0$ tal que

$$
W_{N}=\left(\frac{a_{N}}{b_{N}}-\varepsilon_{N}, \frac{a_{N}}{b_{N}}+\varepsilon_{N}\right) \subset U_{N} \cap\left(\frac{a_{N}}{b_{N}}-\frac{1}{b_{N}^{N}}, \frac{a_{N}}{b_{N}}+\frac{1}{b_{N}^{N}}\right) .
$$

Segue do lema anterior que $Z_{N}=g_{1}\left(W_{N}\right)$ é aberto, contido em $V_{N}$, e $f$ é localmente injetiva em $\alpha$ sobre $W_{N} \times Z_{N}$. Selecionamos $\frac{c_{N}}{d_{N}} \in \mathbb{Q}$ de modo que

$$
\frac{c_{N}}{d_{N}} \in Z_{N} \backslash\left\{\frac{c_{1}}{d_{1}}, \frac{c_{2}}{d_{2}}, \ldots, \frac{c_{N-1}}{d_{N-1}}\right\}
$$

em seguida, selecionamos $u_{N} \in W_{N}$ tal que $f\left(u_{N}, \frac{c_{N}}{d_{N}}\right)=\alpha$.

Observe que,

$$
Z_{N} \cap\left(\frac{c_{N}}{d_{N}}-\frac{1}{d_{N}^{N}}, \frac{c_{N}}{d_{N}}+\frac{1}{d_{N}^{N}}\right)
$$

é aberto, contendo $\frac{c_{N}}{d_{N}}$. Logo, existe $\delta_{N}>0$ tal que

$$
V_{N+1}=\left(\frac{c_{N}}{d_{N}}-\delta_{N}, \frac{c_{N}}{d_{N}}+\delta_{N}\right) \subset Z_{N} \cap\left(\frac{c_{N}}{d_{N}}-\frac{1}{d_{N}^{N}}, \frac{c_{N}}{d_{N}}+\frac{1}{d_{N}^{N}}\right) .
$$


Segue do lema que $U_{N+1}=g_{1}^{-1}\left(V_{N+1}\right)$ é aberto, contido em $W_{N}$, e $f$ é localmente injetiva em $\alpha$ sobre $U_{N+1} \times V_{N+1}$.

Agora, observamos que dado qualquer inteiro $M \geq 1$, para todos os inteiros $m_{1}, m_{2}$ suficientemente grandes,

$$
\left|\frac{a_{m_{1}}}{b_{m_{1}}}-\frac{a_{m_{2}}}{b_{m_{2}}}\right|<\varepsilon_{M} \text { e }\left|\frac{c_{m_{1}}}{d_{m_{1}}}-\frac{c_{m_{2}}}{d_{m_{2}}}\right|<\delta_{M}
$$

Como $\varepsilon_{M} \longrightarrow 0$ e $\delta_{M} \longrightarrow 0$ quando $M \longrightarrow \infty$, segue que as sequências $\frac{a_{m}}{b_{m}}$ e $\frac{c_{m}}{d_{m}}$ são de Cauchy, consequentemente convergem em $\mathbb{R}$. Sejam $\sigma$ e $\tau$ números reais tais que

$$
\lim _{m \longrightarrow \infty} \frac{a_{m}}{b_{m}}=\sigma \mathrm{e} \lim _{m \longrightarrow \infty} \frac{c_{m}}{d_{m}}=\tau,
$$

assim, por construção,

$$
\lim _{m \longrightarrow \infty} u_{m}=\sigma
$$

e, para todo $m \in \mathbb{N}$,

$$
0<\left|\sigma-\frac{a_{m}}{b_{m}}\right|<\frac{1}{b_{m}^{m}} \text { e } 0<\left|\tau-\frac{c_{m}}{d_{m}}\right|<\frac{1}{d_{m}^{m}}
$$

Portanto, ambos $\sigma$ e $\tau$ são números de Liouville.

Finalmente recordamos que, para todo $m, f\left(u_{m}, c_{m} / d_{m}\right)=\alpha$. Como $f$ é contínua e $\lim _{m \longrightarrow \infty} u_{m}=\sigma$ tem-se $f(\sigma, \tau)=\alpha$, o que completa a prova.

O Teorema de decomposição generaliza o Teorema de Erdös, uma vez que a função $f(x, y)=x+y$ satisfaz as hipóteses desse teorema para qualquer $\alpha \in \mathbb{R}$ fixado. Além disso, dada a função $f: \mathbb{R}_{+}^{*} \times((0,1) \cup(1,+\infty)) \longrightarrow \mathbb{R}$, definida por $f(z, w)=w^{1 / z}$, é possível verificar que ela satisfaz as hipóteses do teorema para $x$ positivo diferente de 0 e 1 . Logo, dado $x \in(0,1) \cup(1,+\infty)$, existem $l_{1}$ e $l_{2}$ números de Liouville tais que $l_{2}^{1 / l_{1}}=x$, consequentemente, $x^{l_{1}}$ é um número de Liouville. Em particular, existem números de Liouville $\sigma$ e $\tau$ tais que $e^{\tau}=\sigma$. 
Garantimos, ainda, que a potenciação de dois números de Liouville nem sempre é um número transcendente. De fato, se $\alpha \in \mathbb{R}$ é algébrico maior do que 1 , então $f(x, y)=y^{x}$ é localmente injetiva em $\alpha$ e assim, pelo Teorema de decomposição, existem números de Liouville $\sigma$ e $\tau$ em $\mathbb{R}$ tais que

$$
\sigma^{\tau}=\alpha
$$

Através de argumentos análogos aos utilizados na demostração do Teorema de decomposição, podemos deduzir a seguinte generalização.

Teorema 3.6 (Teorema de decomposição simultânea) Sejam abertos $U, V_{1}, V_{2}, \ldots, V_{N} \subset \mathbb{R}$ e $\alpha_{1}, \alpha_{2}, \ldots, \alpha_{N} \in \mathbb{R}$. Suponha que, para cada $n$, $1 \leq n \leq N, f_{n}: U \times V_{n} \longrightarrow \mathbb{R}$ é contínua, localmente injetiva em $\alpha_{n}$ sobre $U \times V_{n}$ e as funções $\varphi_{n}: U \longrightarrow V_{n}$ e $\psi_{n}: V_{n} \longrightarrow U$, definidas implicitamente por $f_{n}\left(x, \varphi_{n}(x)\right)=\alpha_{n}$ e $f_{n}\left(\psi_{n}(y), y\right)$, são aplicações abertas. Então, existem números de Liouville $\sigma, \tau_{1}, \tau_{2}, \ldots, \tau_{N}$ em $\mathbb{R}$ de modo que

$$
f_{n}\left(\sigma, \tau_{n}\right)=\alpha_{n}
$$

para todo $n=1,2, \ldots, N$.

Considere $\alpha_{1}, \alpha_{2}, \ldots, \alpha_{N}$ números reais diferentes de 0 e 1 . Então, a função $f_{n}(x, y)=y^{1 / x}$ é localmente injetiva em $\alpha_{n}$. Assim, pelo teorema anterior, existem números de Liouville $\sigma, \tau_{1}, \ldots, \tau_{N}$ tais que $\tau_{1}^{1 / \sigma}=\alpha_{1}, \ldots, \tau_{N}^{1 / \sigma}=$ $\alpha_{N}$. Desse modo, podemos afirmar que, dados $\alpha_{1}, \alpha_{2}, \ldots, \alpha_{N}$ números reais diferentes de 0 e 1 , existe um número de Liouville $\sigma$ tal que $\alpha_{1}^{\sigma}, \alpha_{2}^{\sigma}, \ldots, \alpha_{N}^{\sigma}$ são todos números de Liouville.

Neste capítulo, vimos, como consequência do Teorema de decomposição, que nem sempre a potenciação de dois números de Liouville é transcendente. No próximo capítulo, discutiremos sobre potenciação de números transcendentes em que a base pode ser um número de Liouville. 


\section{Capítulo 4}

\section{Potenciação de Transcendentes}

Em 1934, Gelfond e Schneider, independentemente, provaram a transcendência de $\alpha^{\beta}$ quando $\alpha$ é algébrico diferente de 0 e 1 e $\beta$ é algébrico não racional. No capítulo anterior, vimos que dados dois números muito "bem aproximados" por algébricos de grau 1, a potenciação desses números nem sempre é um número transcendente.

Em 1991, Caveny considerou a transcendência de $\alpha^{\beta}$, quando $\alpha$ é "suficientemente bem aproximado por algébricos de grau limitado" e $\beta$ é algébrico de grau pelo menos dois. Neste capítulo, iremos considerar a transcendência de $\alpha^{\beta}$, quando ambos $\alpha$ e $\beta$ são "suficientemente bem aproximados por algébricos de grau limitado". Faremos isso com base no artigo U-numbers and T-numbers: Some Elementary Transcendence and Algebraic Results, publicado em 1993, pela mesma autora.

Inicialmente, apresentaremos algumas definições e resultados importantes para demonstração do teorema principal deste capítulo.

Dados $d \in \mathbb{N}$ e uma função

$$
\begin{aligned}
& \Delta: \mathbb{N} \longrightarrow \mathbb{R}^{+} \\
& T \longmapsto \Delta(T)
\end{aligned}
$$

com $\limsup _{T} \Delta(T)=\infty$, dizemos que um número complexo $\zeta$ é $(d, \Delta(T))$ aproximável, se existe uma sequência infinita $\zeta_{T}$ de números algébricos satis- 
fazendo

$$
\operatorname{deg}\left(\zeta_{T}\right) \leq d \quad, \quad H\left(\zeta_{T}\right) \leq \exp (T) \quad \text { e } \quad 0<\left|\zeta-\zeta_{T}\right| \leq \exp (-\Delta(T))
$$

onde $\operatorname{deg}\left(\zeta_{T}\right)$ e $H\left(\zeta_{T}\right)$ denotam o grau e a altura de $\zeta_{T}$, respectivamente. Sendo que a altura de $\zeta_{T}$ é dada pelo máximo do valor absoluto dos coeficientes do seu polinômio minimal primitivo sobre $\mathbb{Z}$ (isto é, o polinômio primitivo $P(x) \in \mathbb{Z}[x]$ de menor grau, tal que $\left.P\left(\zeta_{T}\right)=0\right)$.

Neste trabalho, iremos considerar números complexos $\zeta$ que são $(d, \Delta(T))$ aproximáveis para alguma $\Delta(T)$ satisfazendo $\limsup _{T \longrightarrow \infty} \frac{\Delta(T)}{T}=\infty$. Tais $\zeta$ são necessariamente transcendentes e são $U$-números na classificação de números complexos Koksma-Mahler (Ver [5]).

Restringindo a uma subsequência de $\left\{\zeta_{T}\right\}$, se necessário, podemos supor que cada aproximação $\zeta_{T}$ satisfaz

$$
\exp (T-1)<H\left(\zeta_{T}\right) \leq \exp (T)
$$

Além disso, podemos escolher um $d$ mínimo tal que cada aproximação tem grau no máximo $d$ e existe uma quantidade infinita de aproximações tendo grau exatamente $d$. Neste caso, dizemos que $d$ é o grau do $U$-número $\zeta$.

Antes da demonstração do teorema, enunciaremos alguns lemas.

Lema 4.1 Sejam v, w números complexos satisfazendo $\left|w-e^{v}\right| \leq \frac{1}{3}\left|e^{v}\right|$. Então, existe uma determinação do logaritmo de $w$ tal que

$$
|\log w-v| \leq \frac{3}{2} \frac{1}{\left|e^{v}\right|}\left|w-e^{v}\right|
$$

Demonstração. Ver [22, p. 450].

Dados $\alpha_{1}, \alpha_{2}, \beta_{0}, \beta_{1}, \beta_{2}$ números algébricos com $\alpha_{1} \alpha_{2} \neq 0$. Consideramos a forma linear $\Lambda=\beta_{0}+\beta_{1} \log \alpha_{1}+\beta_{2} \log \alpha_{2}$.

Sejam $D$ um inteiro positivo e $A_{1}, A_{2}, A, B$ números reais positivos que satisfazem 


$$
\begin{gathered}
D \geq\left[\mathbb{Q}\left(\alpha_{1}, \alpha_{2}, \beta_{0}, \beta_{1}, \beta_{2}\right): \mathbb{Q}\right], \\
A_{j} \geq \max \left\{H\left(\alpha_{j}\right), \exp \left(\left|\log \alpha_{j}\right|\right), \exp (2)\right\}, \quad j \in\{1,2\}, \\
A=\max \left\{A_{1}, A_{2}, e^{e}\right\} \text { e } B=\max \left\{H\left(B_{j}\right), 0 \leq j \leq 2\right\} .
\end{gathered}
$$

Lema 4.2 Se $\Lambda \neq 0$, então

$$
|\Lambda| \geq \exp (-U)
$$

onde $U=c_{1} D^{4} \log A_{1} \log A_{2}(\log B+\log \log A)$ e $c_{1} \leq 2^{73}$.

Demonstração. Ver [20, p. 284].

Observação 4.3 No teorema a seguir, consideramos $\alpha$ e $\beta$ números complexos satisfazendo $\alpha \log \alpha \neq 0$ e $\beta \neq 0$. Além disso, consideramos $d_{0}$ e $d_{1}$ números naturais, com $d_{1} \geq 2$, mínimos tais que cada aproximação tem grau no máximo $d_{0}$ (respectivamente, $d_{1}$ ) e existe uma quantidade infinita de aproximações tendo grau exatamente $d_{0}$ (respectivamente, $d_{1}$ ).

Teorema 4.4 (Caveny) Sejam $\alpha, \beta$ números complexos não-nulos e $d_{0}, d_{1}$ números naturais com $d_{1} \geq 2$. Existe uma constante positiva $C_{2}$ tal que se $\alpha$ é $\left(d_{0}, C_{2} T \log T\right)$-aproximável e $\beta$ é $\left(d_{1}, C_{2} T \exp T\right)$-aproximável, então $\alpha^{\beta}$ é transcendente.

Demonstração. Primeiramente, fixamos $C_{2}$ satisfazendo as hipóteses do teorema, a ser escolhido posteriormente.

Em seguida, sejam $\left\{a_{T_{j}}\right\}$ e $\left\{b_{S_{j}}\right\}$ sequências de boas aproximações para $\alpha$ e $\beta$, respectivamente, de modo que $\operatorname{deg}\left(a_{T_{j}}\right) \geq 1 \mathrm{e} \operatorname{deg}\left(b_{S_{j}}\right) \geq 2, \operatorname{com} a_{T_{j}}$ diferente de 0 e 1 . Sem perda de generalidade, podemos escolher $\left\{T_{j}\right\}_{j \geq 1}$, de modo que $T_{j}>2 T_{j-1}$.

Agora, fixamos $k$ suficientemente grande, a ser escolhido posteriormente. Logo após, escolhemos $l$ de modo que 


$$
T_{l-1}<\exp \left(S_{k}\right) \leq T_{l}
$$

Por uma questão de simplicidade, escrevemos $b=b_{S_{k}}$ e $a=a_{T_{l}}$. Temos

$$
\begin{gathered}
H(a) \leq \exp \left(T_{l}\right), H(b) \leq \exp \left(S_{k}\right) \leq T_{l} \\
|\alpha-a| \leq \exp \left(-C_{2} T_{l} \log \left(T_{l}\right)\right)<\exp \left(-C_{2} T_{l-1} \log \left(T_{l-1}\right)\right)
\end{gathered}
$$

e

$$
|\beta-b| \leq \exp \left(-C_{2} S_{k} \exp \left(S_{k}\right)\right)<\exp \left(-C_{2} T_{l-1} \log \left(T_{l-1}\right)\right)
$$

Vamos supor, por absurdo, que $\alpha^{\beta}$ é algébrico. Consideramos a seguinte forma linear em logaritmos de números algébricos

$$
\Lambda=\log \alpha^{\beta}-b \log a
$$

Note que $\Lambda$ é não nula, pois $\alpha^{\beta}$ é algébrico e, pelo teorema de GelfondSchneider, $a^{b}$ é transcendente. Observe que

$|\Lambda|=|\beta \log \alpha-b \log \alpha+b \log \alpha-b \log a| \leq|\beta-b||\log \alpha|+|b||\log \alpha-\log a|$, com isso, obteremos um limitante superior para $|\Lambda|$.

AFIRMAÇÃO 1: $|\log \alpha-\log a| \leq c_{2}|\alpha-a|$, onde $c_{2}=\frac{3}{2|\alpha|}$.

No Lema 4.1, tome $v=\log \alpha$ e $w=a$. Note que,

$$
|a-\alpha| \leq \frac{1}{3}|\alpha|
$$

a partir de $k$ suficientemente grande, pois $\alpha$ é fixado, $\left\{a_{T_{l}}\right\}$ converge para $\alpha$ e $l$ é escolhido de modo que $\exp \left(S_{k}\right) \leq T_{l}$. Portanto, $v=\log \alpha$ e $w=a$ satisfazem as hipóteses do Lema 4.1.

Assim,

$$
|\log a-\log \alpha| \leq \frac{3}{2} \frac{1}{|\exp (\log \alpha)|}|a-\exp (\log \alpha)|=\frac{3}{2|\alpha|}|\alpha-a| .
$$


E a Afirmação 1 está provada.

Sabendo que $\left(b_{S_{j}}\right)_{j \in \mathbb{N}}$ é limitada (já que converge) e utilizando (4.1), obtemos

$$
1+H(b)=H\left(b_{S_{k}}\right)+1>\exp \left(S_{k}-1\right)+1
$$

e

$$
\exp \left(S_{k}-1\right)+1 \geq\left|b_{S_{k}}\right|=|b|
$$

para $k$ suficientemente grande. Assim,

$$
|b| \leq H(b)+1 \leq T_{l}+T_{l}=2 T_{l}
$$

para $k$ suficientemente grande. Daí

$$
|\Lambda| \leq|\beta-b||\log \alpha|+|b||\log \alpha-\log a|
$$

com

$$
\begin{gathered}
|\log \alpha-\log a| \leq c_{2}|\alpha-a| \leq c_{2} \exp \left(-C_{2} T_{l} \log \left(T_{l}\right)\right) \\
|\beta-b| \leq\left(\exp \left(-C_{2} T_{l-1} \log T_{l-1}\right)\right) \quad \text { e } \quad|b| \leq 2 T_{l} .
\end{gathered}
$$

Em vista disso, obtemos

$$
|\Lambda| \leq\left(\exp \left(-C_{2} T_{l-1} \log T_{l-1}\right)\right)|\log \alpha|+2 T_{l} c_{2} \exp \left(-C_{2} T_{l} \log T_{l}\right)
$$

Note que, para todo $l \geq 1$,

$$
T_{l}^{\frac{1}{C_{2}}}<\left(\frac{T_{l}}{T_{l-1}}\right)^{T_{l}}<\frac{T_{l}^{T_{l}}}{T_{l-1}^{T_{l-1}}},
$$

já que $C_{2}>1$ (a ser escolhida), $T_{l}>2 T_{l-1}$ e $T_{l}^{T_{l}} / T_{l-1}^{T_{l-1}}>T_{l}^{T_{l}} / T_{l-1}^{T_{l}}$. Logo,

$$
T_{l} \leq \frac{T_{l}^{C_{2} T_{l}}}{T_{l-1}^{C_{2} T_{l-1}}}=\exp \left(\log \left(\frac{T_{l}^{C_{2} T_{l}}}{T_{l-1}^{C_{2} T_{l-1}}}\right)\right)=\exp \left(C_{2} T_{l} \log T_{l}-C_{2} T_{l-1} \log T_{l-1}\right) .
$$


Daí,

$$
2 T_{l} c_{2} \exp \left(-C_{2} T_{l} \log T_{l}\right) \leq 2 c_{2} \exp \left(-C_{2} T_{l-1} \log T_{l-1}\right)
$$

Por (4.3),

$|\Lambda| \leq|\log \alpha|\left(\exp \left(-C_{2} T_{l-1} \log T_{l-1}\right)\right)+2 c_{2} \exp \left(-C_{2} T_{l-1} \log T_{l-1}\right)$.

Portanto,

$$
|\Lambda| \leq c_{3} \exp \left(-C_{2} T_{l-1} \log \left(T_{l-1}\right)\right)
$$

$\operatorname{com} c_{3}=2 \max \left\{|\log \alpha|, 2 c_{2}\right\}$.

Assim, para $k$ suficientemente grande, temos

$$
|\Lambda| \leq \exp \left(-\left(\frac{C_{2}}{2}\right) T_{l-1} \log \left(T_{l-1}\right)\right)
$$

Esse é nosso limitante superior para $\Lambda$.

Para obter um limitante inferior, utilizaremos o Lema 4.2. Vamos tomar $\alpha_{1}=\alpha^{\beta}, \alpha_{2}=a, \beta_{0}=0, \beta_{1}=1, \beta_{2}=-b$, em seguida, tomamos $D=c_{4} d_{0} d_{1}$, $B=H(b), A_{1}=c_{5}$ e $A=A_{2}=2 \exp \left(T_{l}\right)$, com $c_{4}$ e $c_{5}$ constantes positivas de modo que as condições do Lema 4.2 sejam satisfeitas. Assim,

$$
\begin{aligned}
|\Lambda| & \geq \exp \left(-c_{1} D^{4} \log A_{1} \log A_{2}(\log B+\log (\log A))\right) \\
& =\exp \left(-c_{1}\left(c_{4} d_{0} d_{1}\right)^{4} \log c_{5} \log \left(2 \exp T_{l}\right)\left(\log H(b)+\log \left(\log \left(2 \exp T_{l}\right)\right)\right)\right.
\end{aligned}
$$

com $c_{1} \leq 2^{73}$. Como $T_{l} \geq H(b)$, temos,

$$
\begin{aligned}
|\Lambda| & \geq \exp \left(-c_{1}\left(c_{4} d_{0} d_{1}\right)^{4} \log c_{5} \log \left(2 \exp T_{l}\right)\left(\log T_{l}+\log \left(\log \left(2 \exp T_{l}\right)\right)\right)\right. \\
& =\exp \left(-c_{1}\left(c_{4} d_{0} d_{1}\right)^{4} \log c_{5}\left(\log 2+T_{l}\right)\left(\left(\log T_{l}\right)+\log \left(\log 2+T_{l}\right)\right)\right) \\
& \geq \exp \left(-c_{1}\left(c_{4} d_{0} d_{1}\right)^{4} \log c_{5}\left(2 T_{l}\right)\left(\left(\log T_{l}\right)+\log \left(2 T_{l}\right)\right)\right) \\
& =\exp \left(-c_{1}\left(c_{4} d_{0} d_{1}\right)^{4} \log c_{5}\left(2 T_{l}\right)\left(\left(\log T_{l}\right)+\left(\log 2+\log T_{l}\right)\right)\right) \\
& \geq \exp \left(-c_{1}\left(c_{4} d_{0} d_{1}\right)^{4} \log c_{5}\left(2 T_{l}\right)\left(3 \log T_{l}\right)\right)
\end{aligned}
$$


Sendo assim, para $k$ suficientemente grande,

$$
|\Lambda| \geq \exp \left(-c_{6}\left(d_{0} d_{1}\right)^{4} T_{l} \log T_{l}\right)
$$

$\operatorname{com} c_{6}=6 c_{1} c_{4}^{4} \log c_{5}$.

Por (4.4) e (4.5), temos,

$$
\exp \left(-\left(\frac{C_{2}}{2}\right) T_{l-1} \log \left(T_{l-1}\right)\right) \geq \exp \left(-c_{6}\left(d_{0} d_{1}\right)^{4} T_{l} \log T_{l}\right)
$$

para $k$ suficientemente grande.

Logo,

$$
C_{2} \leq 2 c_{6}\left(d_{0} d_{1}\right)^{4} \frac{T_{l} \log T_{l}}{T_{l-1} \log \left(T_{l-1}\right)}
$$

Provamos que, se $\alpha$ é $\left(d_{0}, C_{2} T \log T\right)$-aproximável, $\beta$ é $\left(d_{1}, C_{2} T \exp T\right)$ aproximável e $\alpha^{\beta}$ é algébrico, então

$$
C_{2} \leq 2 c_{6}\left(d_{0} d_{1}\right)^{4} \frac{T_{l} \log T_{l}}{T_{l-1} \log \left(T_{l-1}\right)}
$$

Daí, se

$$
C_{2}>2 c_{6}\left(d_{0} d_{1}\right)^{4} \frac{T_{l} \log T_{l}}{T_{l-1} \log \left(T_{l-1}\right)}
$$

$\alpha^{\beta}$ é transcendente.

Note que, se $\alpha$ é real e $d_{0}=1$, obtemos condições suficientes para que a potenciação de números transcendentes, em que a base é um número de Liouville, seja um número transcendente. Observe que, para a demonstração dos teoremas principais dos capítulos 4 e 5, utilizamos o fato de estarmos trabalhando com números muito "bem aproximados" por algébricos, que é uma propriedade bem interessantes dos $U$-números, em particular dos números de Liouville. No capítulo seguinte, voltamos a falar sobre a propriedade $G_{\delta}$ do conjunto dos números de Liouville e mostraremos uma série de resultados decorrentes dessa propriedade. 


\section{Capítulo 5}

\section{Números de Liouville e a Propriedade $G_{\delta}$}

No Capítulo 2, vimos que o conjunto dos números de Liouville é um subconjunto $G_{\delta}$ de $\mathbb{R}$. Neste capítulo, temos o objetivo de explorar um pouco mais essa propriedade de $\mathbb{L}$ e, assim, mostrar outros resultados interessantes.

A proposição seguinte foi provada por Alniaçik e Saias, em [1, p. 426], e será utilizada na demonstração dos resultados da seção 5.1.

Proposição 5.1 Seja I um intervalo de $\mathbb{R}$ com interior não vazio, $G$ um subconjunto $G_{\delta}$ de $\mathbb{R} e\left(f_{n}\right)_{n \geq 0}$ uma sequência de funções definidas em $I$, que são contínuas e NLC. Então

$$
\bigcap_{n \geq 0} f_{n}^{-1}(G)
$$

é um subconjunto $G_{\delta}$ sobre $I$.

Observe que, nas hipóteses da Proposição 5.1, se $\varphi: I \longrightarrow \mathbb{R}$ é tal que $f_{n}=\varphi$, para cada $n \geq 0$, então, $\varphi^{-1}(G)$ é um subconjunto $G_{\delta}$ em $I$, para cada $G$ subconjunto $G_{\delta}$ em $\mathbb{R}$. Isto é, a imagem inversa pela função $\varphi$ de todo subconjunto $G_{\delta}$ em $\mathbb{R}$ é $G_{\delta}$ em $I$. Isso ocorre porque a continuidade de $\varphi$ garante que a imagem inversa dos abertos (da interseção) vão ser subconjuntos abertos de $I$ e a densidade decorre por $\varphi$ ser NLC. 
É importante enfatizar que, este capítulo também baseia-se no artigo $L i$ ouville Numbers and Schanuel's Conjecture, de Kumar, Thangadurai e Waldschmidt.

\subsection{Aplicação da Proposição 5.1 aos números de Liouville}

Nesta seção, utilizaremos a Proposição 5.1 para deduzir alguns resultados sobre números de Liouville.

Teorema 5.2 Seja $\mathcal{E}$ um subconjunto enumerável de $\mathbb{R}$. Então, existe um conjunto não enumerável de números de Liouville $F$ tendo simultaneamente as seguintes propriedades.

(i) Para quaisquer $t \in \mathcal{E}$ e $\xi \in F$, o número $\xi+t$ é um número de Liouville.

(ii) Para quaisquer $t \in \mathcal{E} \backslash\{0\}$ e $\xi \in F$, o número $\xi \cdot t$ é um número de Liouville.

(iii) Sejam $t \in \mathcal{E} \backslash\{0\}$ e $\xi \in F$. Defina indutivamente $\xi_{0}=\xi$ e $\xi_{n}=e^{t \cdot \xi_{n-1}}$, para todo $n \geq 1$. Então, todos os números da sequência $\left(\xi_{n}\right)_{n \geq 0}$ são números de Liouville.

(iv) Para qualquer número racional $r \neq 0$ e qualquer $\xi \in F$, o número $\xi^{r}$ é um número de Liouville.

Demonstração. Construiremos separadamente quatro conjuntos $G_{\delta}$ que satisfaçam cada uma dessas propriedades e, assim, a interseção deles será $G_{\delta}$, em particular, não enumerável.

$(I)$ Definimos, para cada $t \in \mathcal{E}, f_{t}:(0,+\infty) \longrightarrow \mathbb{R}$ por $f(x)=x+t$. Pela Proposição 5.1,

$$
\bigcap_{t \in \mathcal{E}} f_{t}^{-1}(\mathbb{L})
$$

é subconjunto $G_{\delta}$ de $(0,+\infty)$, consequentemente,

$$
\mathbb{L} \cap \bigcap_{t \in \mathcal{E}} f_{t}^{-1}(\mathbb{L})
$$


também é subconjunto $G_{\delta}$ de $(0,+\infty)$ e satisfaz $(i)$.

$(I I)$ Definimos, para cada $t \in \mathcal{E}, g_{t}:(0,+\infty) \longrightarrow \mathbb{R}$ por $g(x)=x \cdot t$. Pela Proposição 5.1,

$$
\bigcap_{t \in \mathcal{E}} g_{t}^{-1}(\mathbb{L})
$$

é subconjunto $G_{\delta}$ de $(0,+\infty)$, consequentemente,

$$
\mathbb{L} \cap \bigcap_{t \in \mathcal{E}} g_{t}^{-1}(\mathbb{L})
$$

também é subconjunto $G_{\delta}$ de $(0,+\infty)$ e satisfaz $(i i)$.

$(I I I)$ Definimos, para cada $t \in \mathcal{E}$, a sequência de funções $h_{n}:(0,+\infty) \longrightarrow \mathbb{R}$ por $h_{0}(x)=x$ e $h_{k}(x)=e^{t \cdot h_{k-1}(x)}$, para $k \geq 1$. Pela Proposição 5.1,

$$
\bigcap_{n \geq 0} h_{n}^{-1}(\mathbb{L})
$$

é $G_{\delta}$ em $(0,+\infty)$. Como $\mathcal{E}$ é enumerável,

$$
\bigcap_{t \in \mathcal{E}} \bigcap_{n \geq 0} h_{n}^{-1}(\mathbb{L})
$$

é $G_{\delta}$ em $(0,+\infty)$ e satisfaz $(i i i)$.

$(I V)$ Definimos, para cada $r \in \mathbb{Q} \backslash\{0\}, \varphi_{r}(x)=x^{r}$. Pela Proposição 5.1,

$$
\bigcap_{r \in \mathbb{Q} \backslash\{0\}} \varphi_{r}^{-1}(\mathbb{L})
$$

é subconjunto $G_{\delta}$ de $(0,+\infty)$, consequentemente,

$$
\mathbb{L} \cap \bigcap_{r \in \mathbb{Q} \backslash\{0\}} \varphi_{r}^{-1}(\mathbb{L})
$$

também é subconjunto $G_{\delta}$ de $(0,+\infty)$ e satisfaz $(i v)$.

Teorema 5.3 Sejam I um intervalo de $\mathbb{R}$ com interior não-vazio e $\left(f_{n}\right)_{n \geq 1}$ uma sequência de funções definidas em I que são contínuas e NLC. Então, existe um subconjunto não enumerável $E$ de $I \cap \mathbb{L}$ tais que $f_{n}(\xi)$ é um número de Liouville para todo $n \geq 1$ e todo $\xi \in E$. 
Demonstração. Definimos $f_{0}: I \longrightarrow \mathbb{R}$ por $f_{0}(x)=x$, para cada $x \in I$, assim $f_{0}$ é contínua e NLC. Pela Proposição 5.1,

$$
E=\bigcap_{n \geq 0} f_{n}^{-1}(\mathbb{L})
$$

é um subconjunto $G_{\delta}$ de $I$, consequentemente, não enumerável. Por fim, observe que $E \subset I \cap \mathbb{L}$ e $f_{n}(E) \subset \mathbb{L}$, para cada $n \geq 0$. Portanto, $f_{n}(\xi) \in \mathbb{L}$, para todo $n \geq 0$ e todo $\xi \in E$.

A seguir, consideramos o caso especial onde todas as $f_{n}$ são as mesmas.

Teorema 5.4 Seja I um intervalo de $\mathbb{R}$ com interior não-vazio e $\varphi: I \longrightarrow \mathbb{R}$ uma aplicação contínua que é NLC. Então, existe um conjunto não enumerável de números de Liouville $\xi \in I$ tais que $\varphi(\xi)$ é um número de Liouville.

Demonstração. Definimos $f_{n}: I \longrightarrow \mathbb{R}$ por $f_{n}(x)=\varphi(x)$, para cada $n \geq 1$. Pelo Teorema 5.3, existe um subconjunto não enumerável $E \subset I \cap \mathbb{L}$ tal que $f_{n}(\xi)$ é um número de Liouville para todo $n \geq 1$ e todo $\xi \in E$. Como, para cada $n \geq 1, f_{n}(x)=\varphi(x)$, segue o resultado.

Exemplos simples de consequências do Teorema 5.4 são obtidos com $I=$ $(0,+\infty)$ e $\varphi(x)=t-x$, que produz o resultado de Erdös (visto no Capítulo 3). Deduzimos também do Teorema 5.4 que qualquer número real positivo $t$ é soma de dois quadrados de números de Liouville, basta considerar $I=(0, \sqrt{t})$ e $\varphi(x)=\sqrt{t-x^{2}}$.

No Capítulo 3, apresentamos uma generalização para o resultado de Erdös utilizando aproximações por racionais. O próximo teorema também generaliza o resultado de Erdös.

Teorema 5.5 Seja $P \in \mathbb{R}[x, y]$ um polinômio irredutivel tal que

$$
\frac{\partial P}{\partial x} \neq 0 e \frac{\partial P}{\partial y} \neq 0 .
$$


Assuma que existem dois intervalos abertos não vazios $I$ e $J$ de $\mathbb{R}$, de modo que, para qualquer $x \in I$, existe $y \in J \operatorname{com} P(x, y)=0$, e, para qualquer $y \in J$ existe $x \in I$ com $P(x, y)=0$. Então, existe uma quantidade não enumerável de pares $(\xi, \eta)$ de números de Liouville em $I \times J$ tais que $P(\xi, \eta)=0$.

Demonstração. Seja $\left(x_{0}, y_{0}\right) \in I \times J$ tal que

$$
\frac{\partial P}{\partial x}\left(x_{0}, y_{0}\right) \neq 0
$$

Pelo Teorema da Função Implícita, existem intervalos abertos não vazios $I_{0}=\left(x_{0}-\delta_{0}, x_{0}+\delta_{0}\right)$ e $J_{0}=\left(y_{0}-\varepsilon_{0}, y_{0}+\varepsilon_{0}\right)$, com $\delta_{0}, \varepsilon_{0}>0$, tais que:

1. $\bar{I}_{0} \times J_{0} \subset I \times J ; \frac{\partial P}{\partial x}(x, y) \neq 0$ para todo $(x, y) \in I_{0} \times J_{0}$

2. Para todo $x \in I_{0}$ existe um único $y=\varphi(x) \in J_{0}$ tal que $P(x, \varphi(x))=0$.

Além disso, a função $\varphi_{0}: I_{0} \longrightarrow J_{0}$ é diferenciável.

Agora, seja $\left(x_{1}, y_{1}\right) \in I_{0} \times J_{0}$ tal que

$$
\frac{\partial P}{\partial y}\left(x_{1}, y_{1}\right) \neq 0
$$

Pelo Teorema da Função Implícita, garantimos a existência de intervalos abertos não vazios $I_{1}=\left(x_{1}-\delta_{1}, x_{1}+\delta_{1}\right) J_{1}=\left(y_{1}-\varepsilon_{1}, y_{1}+\varepsilon_{1}\right)$, com $\delta_{1}, \varepsilon_{1}>0$ tais que:

1. $I_{1} \times \bar{J}_{1} \subset I_{0} \times J_{0} ; \frac{\partial P}{\partial y}(x, y) \neq 0$ para todo $(x, y) \in I_{1} \times J_{1} ;$

2. Para todo $y \in J_{1}$ existe um único $x=\varphi_{1}(y) \in I_{1}$ tal que $P\left(\varphi_{1}(y), x\right)=0$.

Além disso, a função $\varphi_{1}: J_{1} \longrightarrow I_{1}$ é diferenciável.

Definimos $\varphi_{2}: I_{1} \longrightarrow J_{1}, \operatorname{com} \varphi_{2}(x)=\varphi_{0}(x)$, para $x \in I_{1}$. Assim, $\varphi_{2} \circ \varphi_{1}=$ $I d: J_{1} \longrightarrow J_{1}$ e $\varphi_{1} \circ \varphi_{2}=I d: I_{1} \longrightarrow I_{1}$.

Logo, $\varphi_{1}$ e $\varphi_{2}$ são duas funções diferenciáveis, definidas sobre subconjuntos abertos não vazios $J_{1}$ de $J$ e $I_{1}$ de $I$, respectivamente, de modo que $P\left(x, \varphi_{2}(x)\right)=0$ e $P\left(\varphi_{1}(y), y\right)=0$ para $x \in I_{1}$ e $y \in J_{1}$, tais que $\varphi_{2} \circ \varphi_{1}$ é 
a identidade sobre $J_{1}$ e $\varphi_{1} \circ \varphi_{2}$ é a identidade sobre $I_{1}$. Por fim, aplicamos o Teorema 5.4.

O resultado de Erdös sobre $t=\xi+\eta$ para $t \in \mathbb{R}$ segue do Teorema $5.5 \mathrm{com}$ $P(x, y)=x+y-t$. Também o fato de que qualquer número real positivo $t$ é a soma de dois quadrados de números de Liouville segue aplicando o Teorema 5.5 ao polinômio $x^{2}+y^{2}-t$.

Poderíamos deduzir, sob as hipóteses do Teorema 5.5, a existência de um par de números de Liouville $(\xi, \eta)$ com $P(\xi, \eta)=0$ aplicando o Teorema 3.4 com $f(x, y)=P(x, y)$ e $\alpha=0$. Entretanto, com o Teorema 5.5, produzimos uma quantidade não enumerável de soluções.

A seguir, estendemos o Teorema 5.5 para mais de 2 variáveis.

Teorema 5.6 Sejam $m \geq 2$ e $P \in \mathbb{R}\left[x_{1}, \ldots, x_{m}\right]$ um polinômio irredutível tal que

$$
\frac{\partial P}{\partial x_{1}} \neq 0 e \frac{\partial P}{\partial x_{2}} \neq 0
$$

Assuma que existem subconjuntos abertos $I_{i}$ de $\mathbb{R}(i=1, \ldots, m)$ tais que, para qualquer $i \in\{1,2\}$ e qualquer $(m-1)$-upla

$$
\left(x_{1}, \ldots, x_{i-1}, x_{i+1}, \ldots, x_{m}\right) \in I_{1} \times \ldots \times I_{i-1} \times I_{i+1} \times \ldots \times I_{m},
$$

existe $x_{i} \in I_{i}$ tais que $P\left(x_{1}, \ldots, x_{m}\right)=0$. Então, existe uma quantidade não enumerável de uplas $\left(\xi_{1}, \xi_{2}, \ldots, \xi_{m}\right) \in I_{1} \times I_{2} \times \ldots \times I_{m}$ de números de Liouville tais que $P\left(\xi_{1}, \xi_{2}, \ldots, \xi_{m}\right)=0$.

Demonstração. Provaremos o resultado por indução.

O caso $m=2$ segue do Teorema 5.5. Assumimos que o resultado é válido para $m-1$, com $m \geq 3$. Como $\mathbb{L}$ é denso em $\mathbb{R}$, existe uma $(l-2)$-upla de números de Liouville $\left(\xi_{3}, \ldots, \xi_{l}\right) \in I_{3} \times \ldots \times I_{l}$. Seja

$$
P\left(x_{1}, x_{2}, \xi_{3}, \ldots, \xi_{l}\right) \in \mathbb{R}\left[x_{1}, x_{2}\right] .
$$


Por hipótese, dado $x_{1} \in I_{1}$, existe $x_{2} \in I_{2}$ tal que $P\left(x_{1}, x_{2}, \xi_{3}, \ldots, \xi_{l}\right)=0$ e dado $x_{2} \in I_{2}$, existe $x_{1} \in I_{1}$ tal que $P\left(x_{1}, x_{2}, \xi_{3}, \ldots, \xi_{l}\right)=0$. Além disso,

$$
\frac{\partial P}{\partial x_{1}} \neq 0 \text { e } \frac{\partial P}{\partial x_{2}} \neq 0
$$

Sendo assim, pela Proposição 5.5, existe uma quantidade não enumerável de pares $\left(\xi_{1}, \xi_{2}\right)$ de números de Liouville tais que $P\left(\xi_{1}, \xi_{2}, \xi_{3}, \ldots, \xi_{l}\right)=0$. O que encerra a demonstração.

\subsection{Outros teoremas}

A proposição a seguir generaliza a Proposição 5.1 e sua demonstração segue de modo similar à demostração da Proposição 2.20.

Proposição 5.7 Sejam $I, J$ intervalos de $\mathbb{R}$ com interior não vazio, $G$ um subconjunto $G_{\delta}$ de $J$ e $\left(f_{n}\right)_{n \geq 0}$ uma sequência de aplicações, $f_{n}: I \longrightarrow J$, que são contínuos e NLC. Então

$$
\bigcap_{n \geq 0} f_{n}^{-1}(G)
$$

é um subconjunto $G_{\delta}$ sobre $I$.

Como consequência da Proposição 5.7 temos o seguinte teorema.

Teorema 5.8 Seja I um intervalo de $\mathbb{R}$ com interior não vazio e $\varphi: I \longrightarrow$ I um homeomorfismo. Então o conjunto de elementos $\xi$ em I tais que a órbita $\left\{\varphi^{n}(\xi) \mid n \in \mathbb{Z}\right\}$ consiste somente de números de Liouville em I é um subconjunto $G_{\delta}$ de I, consequentemente não enumerável.

Observação 5.9 Se I é um intervalo de $\mathbb{R}$ com interior não vazio, $\varphi: I \longrightarrow I$ um homeomorfismo e $\psi: I \longrightarrow I$ é a sua inversa, definimos, para $n \in \mathbb{Z}$, $\varphi^{n}: I \longrightarrow I$ indutivamente como usual: $\varphi^{0}$ é a identidade, $\varphi^{n}=\varphi^{n-1} \circ \varphi$ para $n \geq 1$, e $\varphi^{-n}=\psi^{n}$ para $n \geq 1$. 
Demonstração do Teorema 5.8. Queremos mostrar que $A=\{\xi \in I \mid$ $\left.\varphi^{n}(\xi) \in \mathbb{L} \cap I, n \in \mathbb{Z}\right\}$ é $G_{\delta}$. Seja,

$$
B=\bigcap_{n \in \mathbb{Z}} f_{n}^{-1}(\mathbb{L} \cap I)
$$

onde $f_{n}: I \longrightarrow I$ é definida por $f_{n}(x)=\varphi^{n}(x)$. Mostraremos que $A=B$. De fato,

$$
B=\bigcap_{n \in \mathbb{Z}} f_{n}^{-1}(\mathbb{L} \cap I)=\bigcap_{n \in \mathbb{Z}} \varphi^{-n}(\mathbb{L} \cap I)=\bigcap_{n \in \mathbb{Z}}\left\{x \in I \mid \varphi^{n}(x) \in \mathbb{L} \cap I\right\}=A .
$$

Observe que $\mathbb{L} \cap I$ é um subconjunto $G_{\delta}$ de $I$, com a topologia induzida. Além disso, $f_{n}$ é um homeomorfismo, para cada $n \in \mathbb{Z}$, consequentemente, é NLC. Pela Proposição 5.7, $f_{n}^{-1}(\mathbb{L} \cap I)$ é $G_{\delta}$ para cada $n \in \mathbb{Z}$. Sendo assim, $\bigcap_{n \in \mathbb{Z}} f_{n}^{-1}(\mathbb{L} \cap I)$ é um subconjunto $G_{\delta}$ de $I$.

Exemplo 5.10 Seja $\varphi:(0,1) \longrightarrow(0,1)$, definida por $\varphi(x)=x^{2}$. Temos que $\varphi$ é um homeomorfismo, logo, pelo Teorema 5.8, existe uma quantidade não enumerável de elementos $\xi \in(0,1)$, tais que

$$
\cdots, \sqrt[8]{\xi}, \sqrt[4]{\xi}, \sqrt{\xi}, \xi, \xi^{2}, \xi^{4}, \xi^{8}, \ldots
$$

são todos números de Liouville.

Teorema 5.11 Seja $F(X, Y) \in \mathbb{Q}[X, Y]$ um polinômio não constante e $t$ um número real. Assuma que existe um conjunto não enumerável de pares de números de Liouville $(\xi, \eta)$ tais que $F(\xi, \eta)=t$. Então, as duas seguintes condições são equivalentes:

(i) $t$ é transcendente.

(ii) Existem dois números de Liouville algebricamente independentes tais que $F(\xi, \eta)=t$.

Para provar esse resultado, utilizaremos o Teorema de Bézout. 
Teorema 5.12 (Teorema de Bézout) Seja $K$ um corpo. Sejam $f(X, Y)$, $g(X, Y)$ dois polinômios em $K[X, Y]$ de graus $n, m \geq 1$. Se $f(X, Y)$ e $g(X, Y)$ não tem fator em comum em $K[X, Y] \backslash K$, então

$$
\#\left\{(x, y) \in K^{2} \mid f(x, y)=0\right\} \cap\left\{(x, y) \in K^{2} \mid g(x, y)=0\right\} \leq n m
$$

Demonstração. Ver [10, p. 71]

Demonstração do Teorema 5.11. Inicialmente, assumimos que $t$ é algébrico. Portanto, existe $P(X) \in \mathbb{Q}[X] \backslash\{0\}$ tal que $P(t)=0$. Para qualquer par de números de Liouville $(\xi, \eta)$ tais que $F(\xi, \eta)=t$, temos $P(F(\xi, \eta))=0$. Note que, $P \circ F \in \mathbb{Q}[X, Y] \backslash\{0\}$, sendo assim, $\xi$ e $\eta$ são algebricamente dependentes. Reciprocamente, assumimos que para qualquer par de números de Liouville $(\xi, \eta)$ tais que $F(\xi, \eta)=t$, os números $\xi$ e $\eta$ são algebricamente dependentes.

AFIRMAÇÃO 1: Existe um polinômio $A(X, Y) \in \mathbb{Q}[X, Y]$ tal que $A(X, Y)$ e $F(X, Y)-t$ tem infinitos zeros em comum.

Por hipótese, existe uma conjunto não enumerável de pares de números de Liouville $(\xi, \eta)$ tais que $F(\xi, \eta)=t$, além disso, estamos supondo que para qualquer par $(\xi, \eta)$, satisfazendo $F(\xi, \eta)=t$, os números $\xi$ e $\eta$ são algebricamente dependentes, sendo assim, para cada um desses pares, existe um $P(X, Y) \in \mathbb{Q}[X, Y]$, tal que $P(\xi, \eta)=0$. Ao supor que cada polinômio em $\mathbb{Q}[X, Y]$ tem no máximo uma quantidade finita de zeros em comum com $F(X, Y)-t$, pela enumerabilidade de $\mathbb{Q}[X, Y]$, obtemos apenas uma quantidade enumerável de pares algebricamente dependentes sobre $\mathbb{Q}$, o que é uma contradição. Portanto, a Afirmação 1 está provada.

AFIRMAÇÃO 2: Para $A(X, Y)$ da Afirmação 1, existe $B(X, Y) \in \overline{\mathbb{Q}}[X, Y]$ irredutivel, tal que $B(X, Y)$ divide $A(X, Y)$ em $\overline{\mathbb{Q}}[X, Y]$ e $B(X, Y)$ divide $F(X, Y)-t$ em $\overline{\mathbb{Q}(t)}[X, Y]$, onde $\overline{\mathbb{Q}}$ denota o fecho algébrico de $\mathbb{Q}$ e $\overline{\mathbb{Q}(t)}$ denota o fecho algébrico de $\mathbb{Q}(t)$. 
Suponha, por absurdo, que $A(X, Y)$ e $F(X, Y)-t$ não tem fator irredutível em comum em $\overline{\mathbb{Q}(t)}[X, Y]$, segue, pelo Teorema 5.12, que $A(X, Y)$ intersecta $F(X, Y)-t$ em, no máximo, uma quantidade finita de pontos. Entretanto, isso contradiz Afirmação 1. Sendo assim, existe $B(t, X, Y) \in \overline{\mathbb{Q}(t)}[X, Y]$ tal que $B(t, X, Y)$ divide $A(X, Y)$ e $F(X, Y)-t$ em $\overline{\mathbb{Q}(t)}[X, Y]$. Como $A(X, Y)$ não depende de $t$, então, $B(t, X, Y)$ também não depende de $t$. Assim, $B(t, X, Y)=$ $B(X, Y)$ divide $A(X, Y)$ em $\overline{\mathbb{Q}}[X, Y]$ e $B(X, Y)$ divide $F(X, Y)-t$ em $\overline{\mathbb{Q}(t)}[X, Y]$. Portanto, a Afirmação 2 está provada.

Vamos supor, por absurdo, que $t$ é transcendente. Assim, pela Afirmação 2, podemos concluir que $F(X, Y)-t=B(X, Y) C(X, Y)$, onde $C \in \overline{\mathbb{Q}}(t)[X, Y]$. O coeficiente de um monômio $X^{i} Y^{j}$ em $C$ é

$$
\left(\frac{\partial^{i+j}}{\partial X^{i} \partial Y^{j}}\right)\left(\frac{F(X, Y)-t}{B(X, Y)}\right)(0,0) .
$$

Note que $C \in \overline{\mathbb{Q}(t)}[X, Y]$ e $C$ tem grau 1 em $t, \operatorname{logo}, C(X, Y)=D(X, Y)+$ $t E(X, Y), \operatorname{com} D$ e $E$ em $\overline{\mathbb{Q}}[X, Y]$. Segue que

$$
\begin{aligned}
F(X, Y)-t & =B(X, Y)[D(X, Y)+t E(X, Y)] \\
& =B(X, Y) D(X, Y)+t B(X, Y) E(X, Y)
\end{aligned}
$$

que implica

$$
B(X, Y) E(X, Y)=-1
$$

Logo, o grau de $B(X, Y)$ é 0 e, consequentemente, $B(X, Y)$ não é irredutível, o que contradiz a Afirmação 2. Portanto, $t$ é algébrico. 


\section{Referências Bibliográficas}

[1] ALNIAÇIK, K. and SAIAS, E., Une remarque sur les $G_{\delta}$-denses, Archiv der Mathematik, 62 (1994), no. 5, 425-426.

[2] AYOUB, R., Euler and the zeta function, Amer. Math Monthly, 81 (1974), 1067-1086.

[3] BURGER, E., On Liouville decompositions in local fields, Proceedings of the American Mathematical Society, 124 (1996), no. 11, 3305-3310.

[4] CAVENY, D., U-numbers and T-numbers: Some elementary transcendence and algebraic independence results, Number theory with an emphasis on the Markoff spectrum, 147 (1993), 43-52.

[5] CAVENY, D. and TUBBS, R., The arithmetic of well-approximated numbers, Number theory with an emphasis on the Markoff spectrum, 147 (1993), 53-59.

[6] Cheng, C.; Dietel, B.; Herblot, M.; HUANG, J.; KRIEGER, H.;MARQUES, D.; MASON, J.; MEREB, M. and WILSON, S. R., Some consequences of Schanuel's Conjecture, J. Number Theory, 129 (2009), 1464-1467.

[7] ERDÖS, P., Representations of real numbers as sums and products of Liouville numbers, Michigan Math. J., 9 (1962), 59-60.

[8] FEL'DMAN, N. I. and NESTERENKO, Y. V., Number Theory IV: Transcendental Numbers, New York: Springer, 1998. Volume 44. 
[9] KUMAR, K. S., THANGADURAI, R. and WALDSCHMIDT, M., Liouville numbers and Schanuel's Conjecture, Archiv der Mathematik, 102 (2014), no. 1, 59-70.

[10] LEQUAIN, Y. and GARCIA, A., Álgebra: Um Curso de Introdução, Rio de Janeiro: IMPA, 1988.

[11] LIMA, E. L., Curso de Análise, 14 Ed., Rio de Janeiro: IMPA, 2013. Volume 1.

[12] LIMA, E. L., Elementos de Topologia Geral, Rio de Janeiro: Editora SBM, 2009.

[13] LIOUVILLE, J., Remarques relatives à des classes très-étendues de quantités dont la valeur n'est ni algébrique, ni même réductible à des irrationnelles algébriques, C. R. Acad. Sci. Paris, 18 (1844), 883-885.

[14] LIOUVILLE, J., Nouvelle démonstration du'n théorème sur irrationnelles algébriques inséré dans le compte rendu de la dernière séance, C. R. Acad. Sci. Paris, 18 (1844), 910-911.

[15] LIOUVILLE, J., Sur des classes très-étendues de quantités dont la valeur n'est ni algébrique, ni même réductible à des irrationnelles algébriques, Journal de mathématiques pures et appliquées, 16 (1851), no. 1, 133-142.

[16] MAILLET, E., Introduction à la théorie des nombres transcendants et des propriétés arithmétiques des fonctions, Paris: Gauthier-Villars, 1906.

[17] MAHLER, K., Some suggestions for further research, Bulletin of the Australian Mathematical Society, 29 (1984), no. 1, 101-108.

[18] MARQUES, D., Teoria dos Números Transcendentes, 1 ed., Rio de Janeiro: SBM, 2013.

[19] MARQUES, D., Alguns resultados que geram números transcendentes, Dissertação de Mestrado, Universidade Federal do Ceará, Brasil, 2007. 
[20] PHILIPPON, P. and WALDSCHMIDT, M., Lower bounds for linear forms in logarithms, New advances in transcendence theory, (1988), 280312.

[21] RIBEnBOIM, P., My Numbers, My Friends: Popular Lectures on Number Theory, Springer-Verlag, 2000.

[22] WALDSCHMIDT, M., Transcendence measures for exponentials and logarithms. Journal of the Australian Mathematical Society (Series A), 25 (1978), no. 4, 445-465.

[23] ZARISKI, O. and SAMUEL, P., Commutative Algebra, New Jersey: D. Van Nostrand Company, Inc., 1958. Volume 1. 